\title{
Restoring fertility in yeast hybrids: Breeding and quantitative genetics of beneficial traits
}

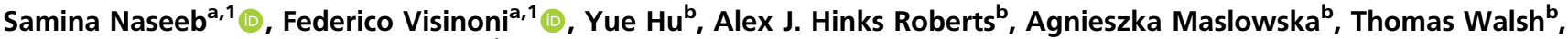 \\ Katherine A. Smart ${ }^{c}$, Edward J. Louis ${ }^{b, 2}{ }^{\circ}$, and Daniela Delneri ${ }^{a, 2}(1)$ \\ ${ }^{a}$ Manchester Institute of Biotechnology, University of Manchester, Manchester M1 7DN, United Kingdom; ${ }^{b}$ Department of Genetics and Genome Biology، \\ University of Leicester, Leicester LE1 7RH, United Kingdom; and 'Department of Chemical Engineering and Biotechnology, University of Cambridge, \\ Cambridge CB3 OAS, United Kingdom
}

Edited by Chris Todd Hittinger, University of Wisconsin-Madison, Madison, WI, and accepted by Editorial Board Member Jasper Rine July 29, 2021 (received for review January 20, 2021)

Hybrids between species can harbor a combination of beneficial traits from each parent and may exhibit hybrid vigor, more readily adapting to new harsher environments. Interspecies hybrids are also sterile and therefore an evolutionary dead end unless fertility is restored, usually via auto-polyploidisation events. In the Saccharomyces genus, hybrids are readily found in nature and in industrial settings, where they have adapted to severe fermentative conditions. Due to their hybrid sterility, the development of new commercial yeast strains has so far been primarily conducted via selection methods rather than via further breeding. In this study, we overcame infertility by creating tetraploid intermediates of Saccharomyces interspecies hybrids to allow continuous multigenerational breeding. We incorporated nuclear and mitochondrial genetic diversity within each parental species, allowing for quantitative genetic analysis of traits exhibited by the hybrids and for nuclear-mitochondrial interactions to be assessed. Using pooled F12 generation segregants of different hybrids with extreme phenotype distributions, we identified quantitative trait loci (QTLs) for tolerance to high and low temperatures, high sugar concentration, high ethanol concentration, and acetic acid levels. We identified QTLs that are species specific, that are shared between species, as well as hybrid specific, in which the variants do not exhibit phenotypic differences in the original parental species. Moreover, we could distinguish between mitochondria-type-dependent and independent traits. This study tackles the complexity of the genetic interactions and traits in hybrid species, bringing hybrids into the realm of full genetic analysis of diploid species, and paves the road for the biotechnological exploitation of yeast biodiversity.

hybrids | QTL | yeast | breeding

$\mathbf{H}$ bridization is important evolutionarily as well as industrially, as it may offer advantageous gene combinations and traits of interest to the newly formed hybrids. Hybrids between species are commonly found in both natural and domestic situations with as many as $25 \%$ of plant species and $10 \%$ of animal species hybridizing naturally (1). The genus Saccharomyces encompasses eight species, included the newly discovered Saccharomyces jurei (2, 3 ), which all readily hybridize among them. In the Saccharomyces yeasts, there are many examples of hybrids from both natural (wild) as well as fermentation sources, and indeed, as many as $10 \%$ of all yeast isolates are hybrids (4-6). The stringent condition of beer and wine fermentations in particular represent a fertile ground for hybrids, influencing their creation, stabilization, and phenotypic and genetic makeup $(7,8)$. Indeed, Saccharomyces pastorianus (synonym Saccharomyces carlsbergensis), a Saccharomyces cerevisiae/Saccharomyces eubayanus hybrid, has been employed in beermaking since the 15 th century $(9,10)$. Saccharomyces cerevisiae/Saccharomyces kudriavzevii hybrids have also been isolated from wine, beer, and cider fermentations along with $S$. cerevisiae/Saccharomyces uvarum hybrids and the triple hybrid between $S$. cerevisiae, $S$. uvarum, and $S$. kudriavzevii as well as $S$. cerevisiae, $S$. uvarum, and $S$. eubayanus (11, 12). Moreover, examples of hybrids between the closely related species $S$. cerevisiae and Saccharomyces paradoxus have been isolated from wild environments (13). Interspecies hybrids of Saccharomyces species have therefore been used as model organisms for studying natural evolution, speciation, and fitness adaptation to different environments.

Recently, much work has gone into the generation of de novo yeast hybrids, exploiting their potential for the production of biofuels (14), brewing $(15,16)$, and winemaking (17). Interspecies hybrids are not only selected for their capability to combine the advantageous traits of the parent strains, as the genomes from both parents undergo chromosomal rearrangements, mutations, widespread transcriptional changes $(18,19)$, and gene loss and gene duplications, which also impact the nature of protein complexes formed (20). Hence, new and improved phenotypes can arise thanks to heterosis or hybrid vigor (21).

A great deal has been learned on the acquired properties of particular hybrids from comparative genomics and molecular studies. However, to date, no thorough genome-wide analysis of genetic contributions to traits of industrial interest has been possible. The sterility of the interspecies yeast hybrids is in fact hindering the application of both predictive quantitative approaches and any attempt of strain improvement via breeding. A

\section{Significance}

Interspecies hybrids, for which mules are a common example, are sterile and therefore an evolutionary dead end. Hybrid sterility has been an obstacle to classical genetic analysis, predictive quantitative approaches, and attempts at strain improvement via breeding. Here, we overcame infertility by creating hybrid tetraploids of yeast species to allow continuous multigenerational breeding. Thus, by exploiting interspecific genetic diversity, we were able to create an unprecedented number of meiotic progenies with different combinations of traits. We showed that the offspring of different hybrids have extreme phenotypes, identified quantitative trait loci (QTLs) dependent of the mitochondria, and discovered QTLs that are uniquely generated in hybrids and for which the allelic variation has no phenotypic consequences in the parental species.

Author contributions: E.J.L. and D.D. designed research; S.N., F.V., A.J.H.R., and A.M. performed research; S.N., F.V., Y.H., A.J.H.R., A.M., T.W., K.A.S., E.J.L., and D.D. analyzed data; and S.N., F.V., Y.H., E.J.L., and D.D. wrote the paper.

The authors declare no competing interest.

This article is a PNAS Direct Submission. C.T.H. is a guest editor invited by the Editorial Board.

This open access article is distributed under Creative Commons Attribution-NonCommercialNoDerivatives License 4.0 (CC BY-NC-ND).

${ }^{1}$ S.N. and F.V. contributed equally to this work

${ }^{2}$ To whom correspondence may be addressed. Email: d.delneri@manchester.ac.uk or ejl21@le.ac.uk.

This article contains supporting information online at https://www.pnas.org/lookup/suppl/ doi:10.1073/pnas.2101242118/-/DCSupplemental.

Published September 13, 2021 
previous study by Greig et al. (22) showed that sterility of interspecies yeast hybrids can be overcome by creating tetraploids, using a mating type locus (MAT) deletion strategy. The engineered tetraploid hybrids were able to produce viable diploid progeny after undergoing meiosis (22). Another study by Schwartz et al. (23) demonstrated the ability to mate a hybrid for quantitative trait loci (QTL) analysis using expression of $H O$ to switch a diploid nonmater to a mater. QTL mapping in particular has shown to be a powerful tool for understanding the genetic basis of various complex traits and has been similarly applied in industrial and medical applications $(24,25)$. Nonetheless, QTL analysis and understanding of hybrid genetics remains poor and limited to a single generation of meiosis in these studies, due to the sterility of Saccharomyces hybrids.

In this study, we used two different approaches to bring de novo yeast hybrids into the realm of genetic analysis and improvement by breeding. A large set of de novo hybrids was engineered by crossing geographically distinct Saccharomyces strains of different species. We were able to generate genetically and phenotypically diverse populations through multiple rounds of interbreeding. Diploid F2 and F12 progeny were phenotyped for several traits, and two approaches toward genotyping pools of phenotypic extremes were used to map genetic variation in both parental genomes responsible for the phenotypes selected. For three sets of hybrids, F12 diploid hybrid progeny were arrayed and phenotyped under several different conditions. For each hybrid, two versions were assessed, one each with each parental species mitochondrion. The top 20 and bottom 20 from an array of 384 were pooled for sequencing and the frequencies of segregating single nucleotide polymorphism (SNP) sites in the two parental species genomes were compared to identify QTLs involved in the traits. For one of these hybrids (two mitochondrial versions), strong selection was applied to a population of $10^{8} \mathrm{~F} 12$ progeny resulting in fewer than $10^{6}$ survivors whose pooled genomic DNA was compared to the pool prior to selection, for several conditions. We demonstrate Saccharomyces hybrids are now amenable to all the tools available for yeast, including breeding and utilization of the vast genetic diversity available. We show that there are QTLs both unique to one parent species or shared by both, QTLsdependent and -independent of the mitochondrial origin, and QTLs that are specific to the hybrid and not to the parent species where the variants are segregating.

\section{Results}

Construction of Tetraploid Yeast Hybrids from a Variety of Parental Strains Leads to Restoration of Fertility. We constructed yeast hybrid tetraploid lines combining four different genomes of strains coming from two separate species belonging to the Saccharomyces genus. To construct such fertile tetraploid hybrids, we employed two different strategies (Fig. 1). First interspecies diploid hybrids were constructed using stable haploids after deletion of $H O$ (five for $S$. cerevisiae/S. jurei and 10 for $S$. cerevisiae/S. kudriavzevii, SI Appendix, Table S1), followed by reciprocal deletion of the MAT locus, and subsequent crossing of the two diploid mater hybrids. In the second strategy, intraspecies diploid strains were first constructed by crossing diverged populations of the same species, again using stable haploids after deletion of $H O(10 \mathrm{~S}$. cerevisiae, six $S$. uvarum, and two $S$. eubayanus intraspecies crossings, $S I$ Appendix, Table S2), followed by reciprocal deletion of the MAT locus and subsequent hybridization of the two diploid maters. Tetraploid lines of $S$. cerevisiae/S. kudriavzevii $(\mathrm{Sc} / \mathrm{Sk})$ and $S$. cerevisiae/S. jurei $(\mathrm{Sc} / \mathrm{Sj})$ hybrids were created with this first strategy while $S$. cerevisiae/S. uvarum, and $S$. cerevisiae/S. eubayanus were constructed using the second approach.

Hybrids between Saccharomyces species are homoplasmic and tend to carry mitochondrial DNA from only one parent. The natural hybrid $S$. pastorianus (hybridized from $S$. cerevisiae and $S$. eubayanus) carries the $S$. eubayanus mitochondria $(26,27)$, while other industrial hybrids of Saccharomyces species used in wine and cider production have retained $S$. cerevisiae mitochondrial genome (28). It has also recently been shown that the type of mitochondria inherited affects the phenotype (29-31) and the transcriptional network (18) in hybrids. Therefore, here, each of the tetraploid hybrids constructed were also selected for different mitotypes. Throughout the paper, in the hybrid nomenclature, a subscripted $\mathrm{m}$ following the initial of the species represents the particular mitochondria inheritance of that hybrid (i.e., $\mathrm{Sc}$ the tetraploid hybrid containing Sc mitochondria, and $\mathrm{Sc} / \mathrm{Sj}$ is the tetraploid hybrid containing $\mathrm{Sj}$ mitochondria).

A total of 226 tetraploid hybrids were created, and each hybrid had four unique parental strains and a unique mitochondrion contributing to the genome (Dataset S1). All the constructed tetraploids were fertile as they had a homologous set of chromosomes to align in meiosis. While diploid hybrids between species of Saccharomyces genus are reproductively isolated $(3,4)$, the tetraploid hybrids constructed here were fertile and exhibited spore viability as high as $98 \%$ (SI Appendix, Table S3) as has previously been reported for tetraploids (22). Ultimately, the diploid F1 spores obtained from the tetraploid hybrids were sequentially randomly mated and sporulated 11 times until the F12 generation (Fig. 1). From the F12 generation around 384 spores for each were isolated for further studies.

Meiotic Offspring of Tetraploid Hybrids Exhibit Broad Phenotypic Diversity. Intercrossing different populations of Saccharomyces species over many generations reduces linkage disequilibrium by increasing recombination. To assess whether the fitness traits are associated with genetic linkage, we assessed the phenotypic landscape of F1 and F12 diploid generations in up to 12 conditions encompassing different growth temperatures, carbon sources, and stressors. An example of phenotypic divergence between F1 diploid segregants of $\mathrm{Sc} / \mathrm{Sj}\left(\mathrm{Sc}^{\mathrm{OS} 3} / \mathrm{Sj}^{\mathrm{D} 5088} / \mathrm{Sc}^{\mathrm{OS104}} / \mathrm{Sj}^{\mathrm{D} 5095}\right)$ and $\mathrm{Sc} / \mathrm{Sk}$ $\left(\mathrm{Sc}^{\mathrm{OS} 253} / \mathrm{Sk}^{\mathrm{OS} 575} / \mathrm{Sc}^{\mathrm{OS} 104} / \mathrm{Sk}^{\mathrm{IFO} 1802}\right)$ tetraploids harboring different mitotypes is reported in SI Appendix, Fig. S1. Significant fitness differences were seen in all the segregant lines with a dispersion up to 0.33 (quartile coefficient of dispersion, SI Appendix, Table $\mathrm{S} 4)$, with some progeny being fitter than any of the parents (SI Appendix, Fig. S2) and some being less fit (transgressive variation) in virtually all cases.

When colony size is normalized within each specific condition, to allow the teasing apart of fitness differences between spores derived from the same tetraploid line, F12 segregants of $\mathrm{Sc}_{\mathrm{m}} / \mathrm{Sj}$, $\mathrm{Sc} / \mathrm{Sj}_{\mathrm{m}}, \mathrm{Sc}_{\mathrm{m}} / \mathrm{Sk}$, and $\mathrm{Sc} / \mathrm{Sk}_{\mathrm{m}}$ again exhibited a large phenotypic variation in all the tested conditions (Fig. 2).

Given that the tetraploid lines are composed of $S$. cerevisiae combined with genomes of other Saccharomyces species with different levels of phylogenetic distance, we investigated whether such differences in genome divergence have an impact on phenotypic plasticity in any given condition. By analyzing the unnormalized fitness data, to tease apart differences in the colony size range between progeny from separate tetraploid lines, no striking differences were found in either range or dispersion between different hybrids (SI Appendix, Fig. S3). Therefore, the different levels of divergence of the genomes present did not impact significantly on phenotypic range and plasticity in the progeny in the hybrid lines generated.

Phenotypic Diversity of Tetraploid Hybrids is Underpinned by the Presence of QTLs. To identify the genetic basis underlying the observed phenotypic diversity, we performed QTL analysis on selected segregant pools of $\mathrm{Sc} / \mathrm{Sj}, \mathrm{Sc} / \mathrm{Sk}, \mathrm{Sc} / \mathrm{Se}$, and $\mathrm{Sc} / \mathrm{Su}$ hybrids. Two different methods for QTL analysis were employed. The Multipool technique, pioneered by ref. 32, was used to analyze the F12 generation of $\mathrm{Sc} / \mathrm{Sj}, \mathrm{Sc} / \mathrm{Sk}$, and in $\mathrm{Sc} / \mathrm{Se}$ hybrids, while the Pooled Selection method, or bulk segregant analysis $(33,34)$, was applied to $\mathrm{Sc} / \mathrm{Se}$ and $\mathrm{Sc}_{\mathrm{m}} / \mathrm{Su}$ hybrids. Both approaches proved 
Tetraploid hybrids constructed from inter-species diploids

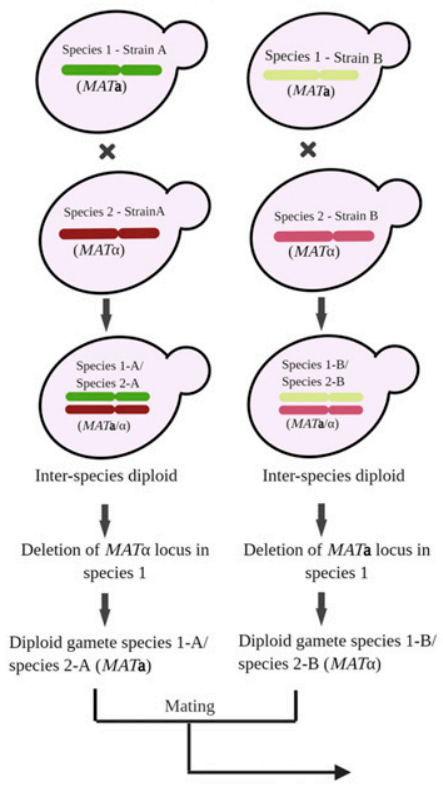

Tetraploid hybrids constructed from intra-species diploids

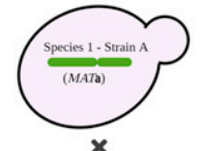

$x$

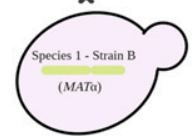

$\downarrow$
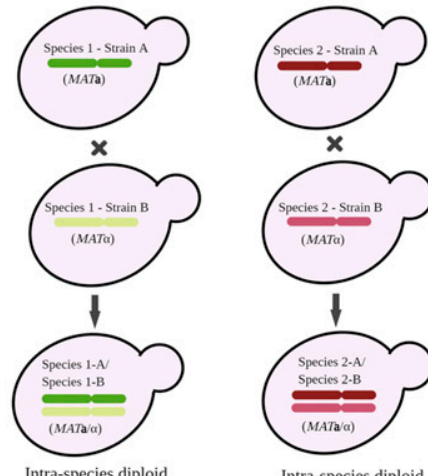

$x$

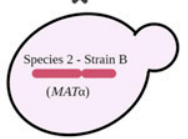

1

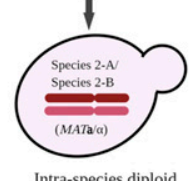

$\downarrow$

Deletion of MATa locus in Deletion of MATa locus in

species $1 \quad$ species 2

$\downarrow$

Diploid gamete species 1-A/

species 1-B (MATa)

Diploid gamete species 2-A
species 2-B (MAT $)$

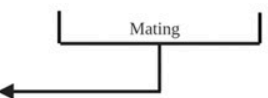

F1 progeny (mater diploids). All progenies will be MATa and MATa
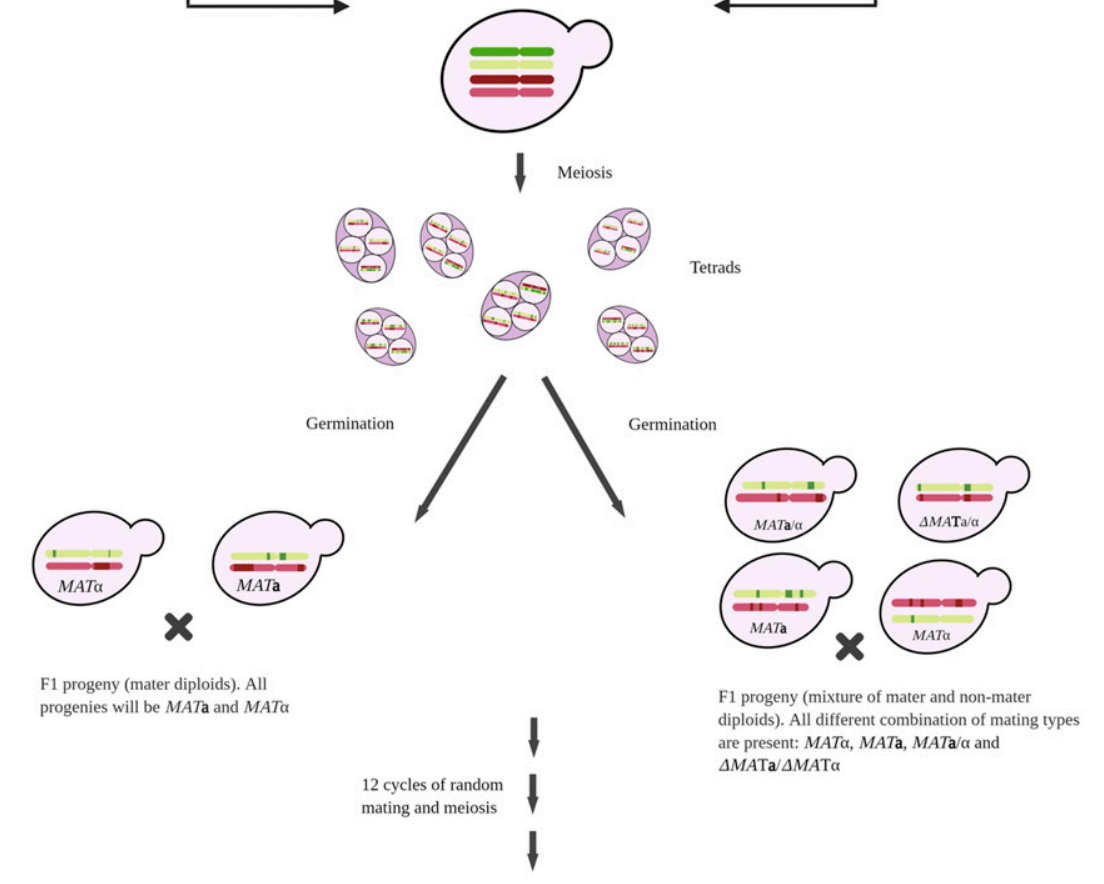

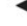
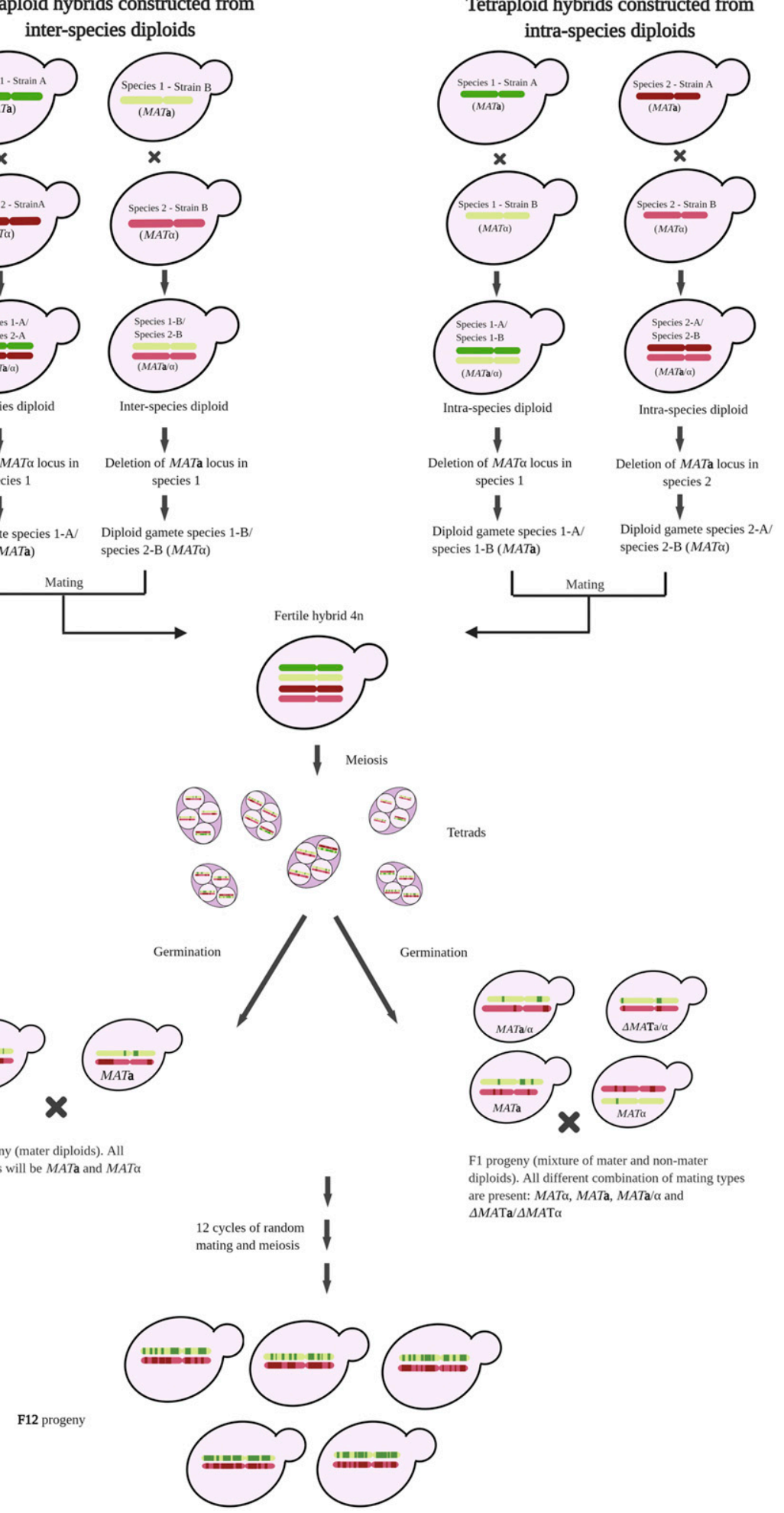

Fig. 1. Construction of fertile hybrids. The tetraploid hybrids were constructed using two different strategies. In the first strategy, two different Saccharomyces species were crossed to obtain $2 \mathrm{n}$ interspecies diploid hybrids (species 1 and species 2 are represented in shade of green and red, respectively). Two different such hybrids were made to behave as gametes by deleting either the MATa or $\alpha$ locus in one species and subsequently crossed to make the fertile tetraploid hybrid $(4 n)$. In the second strategy, two diverged populations (A and $B$ ) of the same species were crossed to construct the $2 n$ intraspecies strains. Two different intraspecies lines were also made to behave as gametes by deleting the MATa from species 1 and $M A T \alpha$ from species 2 , and subsequently, these were crossed to construct the $4 \mathrm{n}$ hybrids. The tetraploid hybrids were sporulated and germinated to obtain the diploid $\mathrm{F} 1$ progeny which were randomly mated and sporulated several times until a F12 generation with a high level of scrambled genomes. 
A

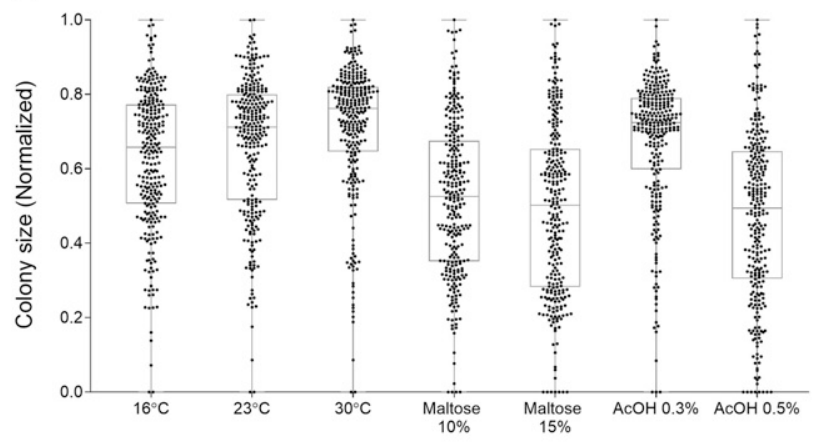

C

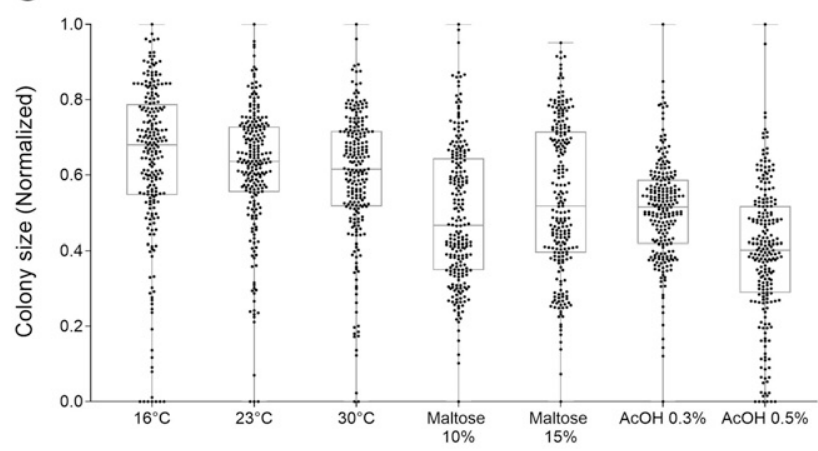

E

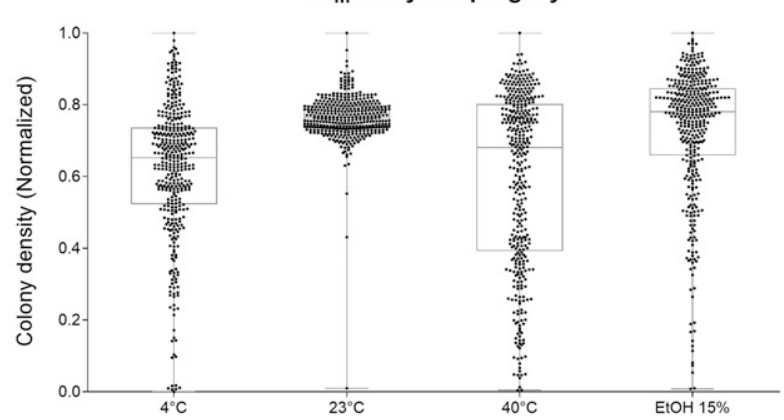

B

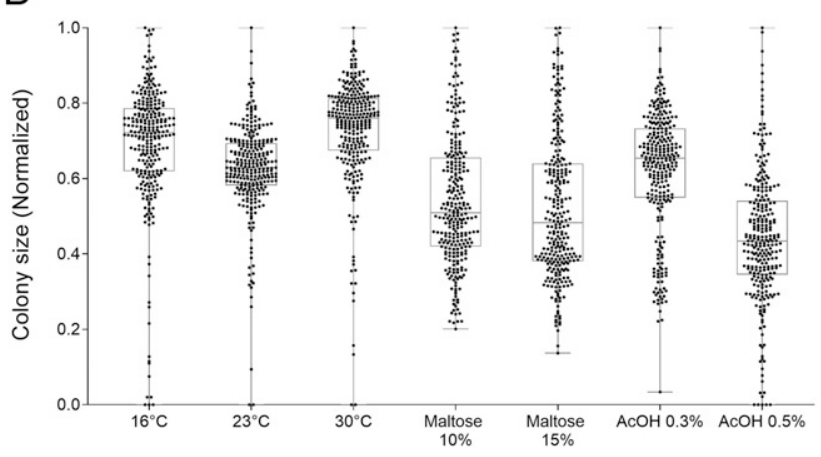

D

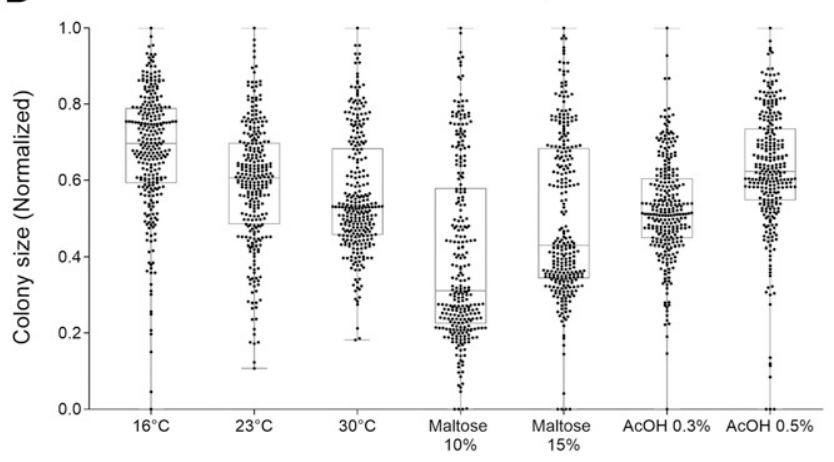

$\mathrm{F}$

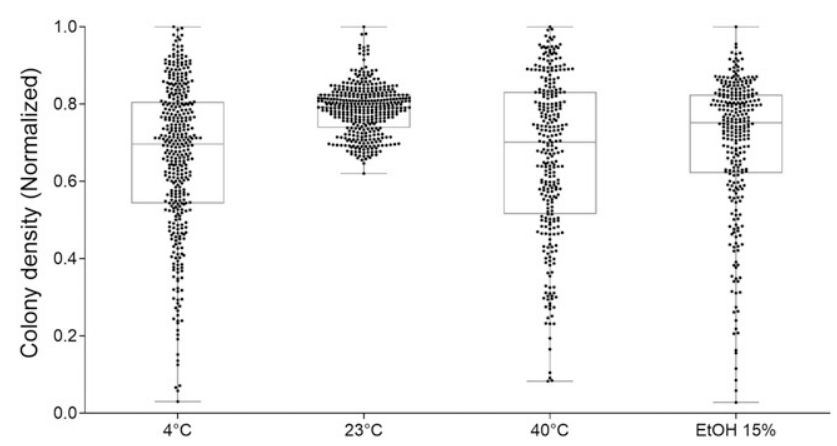

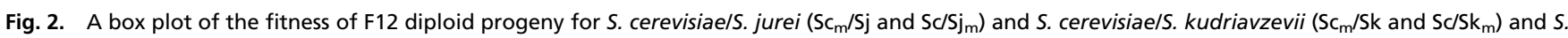
cerevisiae/S. eubayanus ( $\mathrm{Sc}_{\mathrm{m}} / \mathrm{Se}$ and $\mathrm{Sc} / \mathrm{Se} \mathrm{m}_{\mathrm{m}}$ ) hybrids. For $\mathrm{Sc} \mathrm{c}_{\mathrm{m}} / \mathrm{Sj}(A), \mathrm{Sc} / \mathrm{Sj} \mathrm{m}_{\mathrm{m}}(B), \mathrm{Sc}_{\mathrm{m}} / \mathrm{Sk}(C)$, and $\mathrm{Sc} / \mathrm{Sk}_{\mathrm{m}}(D)$, the normalized colony size was used as proxy of fitness (see Materials and Methods) and was scored in YPD at different temperatures $16{ }^{\circ} \mathrm{C}, 23{ }^{\circ} \mathrm{C}$, and $30{ }^{\circ} \mathrm{C}$ in YP- Maltose (10\% and $15 \%$ ) and in YPD with $0.3 \%$ and $0.5 \%$ acetic acid $(\mathrm{AcOH})$. For $\mathrm{Sc}_{\mathrm{m}} / \mathrm{Se}(E)$ and $\mathrm{Sc} / \mathrm{Se}_{\mathrm{m}}(F)$, the colony optical density was used as proxy of fitness (see Materials and Methods) and was scored in YPD at different temperature $4{ }^{\circ} \mathrm{C}, 23{ }^{\circ} \mathrm{C}$, and $40^{\circ} \mathrm{C}$ and in YPD with $15 \%$ Ethanol (EtOH). Each black dot represents a different F12 diploid hybrid. The upper and lower error bars represent the minimum and maximum values.

successful in mapping QTL regions in a variety of conditions for all hybrids; however, a higher number of QTLs and more consistent results across the conditions tested were obtained using the Multipool approach (SI Appendix, Table S5). A comprehensive list of the QTL intervals mapped, including coordinates of the regions, logarithm of the odds (LOD) scores, and gene content is presented in Dataset S2.

For the Multipool, the top 20 and bottom 20 individual of 384 arrayed were pooled for each condition for comparison. $\mathrm{Sc} / \mathrm{Sj}$ and $\mathrm{Sc} / \mathrm{Sk}$ hybrids were selected at low temperature $\left(16^{\circ} \mathrm{C}\right)$, in $15 \%$ maltose, and in $0.3 \%$ acetic acid, while Se/Sc hybrids were selected in $10 \%$ ethanol and low and high temperatures (4 and $40{ }^{\circ} \mathrm{C}$ ). Such conditions are relevant to fermentation industries. Low temperature is required for the storage of brewing yeast and for fermentation of lager beer. Maltose is one of the key wort sugars, and the high concentration of this sugar mimics the osmotic pressure exerted upon yeast in high gravity wort (35). Acetic acid is found in grape must, and wine-producing yeasts are known to require the resistance to this stress $(35,36)$. Ethanol is the major stressor for both the production of fermented beverages and bioethanol (35).

From segregants generated from the tetraploids $\mathrm{Sc}_{\mathrm{m}} / \mathrm{Sj}$ and $\mathrm{Sc} /$ $\mathrm{Sj}_{\mathrm{m}}$, a total of 56 QTLs were identified in the $S$. cerevisiae genomes with an average length of $19.4 \mathrm{~kb}$ (SI Appendix, Table S6). Despite the high similarity between the two $S$. jurei parental strains (2), we were able to map 62 QTL regions in the genome of this species. However, with an average length of $35.5 \mathrm{~kb}$, the QTL mapping intervals were the longest observed in the hybrids 
generated due to the lower density of segregating markers in the two genomes of this species.

An even higher number of QTLs was detected in the progeny of $\mathrm{Sc}_{\mathrm{m}} / \mathrm{Sk}$ and $\mathrm{Sc} / \mathrm{Sk}_{\mathrm{m}}$ tetraploids with up to 155 and 128 regions mapped in $S$. cerevisiae and $S$. kudriavzevii, respectively (SI Appendix, Table S6). Here, the QTL intervals were narrower than those seen in the $S$. jurei genome, with only $15.6 \mathrm{~kb}$ average length on $S$. cerevisiae alleles and $18.6 \mathrm{~kb}$ on $S$. kudriavzevii ones, as there is a higher density of segregating markers in these genomes.

Similar results were obtained in $\mathrm{Sc}_{\mathrm{m}} / \mathrm{Se}$ and $\mathrm{Sc} / \mathrm{Se}_{\mathrm{m}}$ hybrids analyzed for their fitness in high ethanol concentration and at high and low temperatures $\left(40\right.$ and $\left.4{ }^{\circ} \mathrm{C}\right)($ SI Appendix, Table S6). Here, we were able to identify 111 and 64 QTLs regions in $S$. cerevisiae and $S$. eubayanus, respectively, with an average length of $18.5 \mathrm{~kb}$ and $21.82 \mathrm{~kb}$.

A total of 28 genes mapped in different QTLs in $\mathrm{Sc} / \mathrm{Sj}$ and $\mathrm{Sc} /$ Sk hybrids were classified as potential causal genes, as their role in the selection condition was already confirmed by previous published work (37) (SI Appendix, Table S7). For example, one of the acetic acid QTL detected in $S$. cerevisiae chromosome III (52 to $97 \mathrm{~kb}$ ) in $\mathrm{Sc}_{\mathrm{m}} \mathrm{Sj}$ hybrids contains variant alleles of $L E U 2$. The gene encodes for a $\beta$-isopropyl-malate dehydrogenase, and null mutants are reported as sensitive to acetic acid while its overexpression increase acetic acid resistance (38).

Among the genes identified, a total of 43 genes with segregating alleles found in low-temperature QTLs were previously identified in large-scale competition studies carried out in $S$. cerevisiae at $16{ }^{\circ} \mathrm{C}$, with an additional five being described as cold favoring by thermodynamic model predictions (SI Appendix, Table S7) (39).

Thanks to the abundance of data on heat and ethanol sensitivity in $S$. cerevisiae $(34,40-42)$, a high number of potential causal genes with segregating variation were identified in the QTL regions of $\mathrm{Sc} / \mathrm{Se}$ hybrids. Thus, we were able to identify up to 38 genes in the 44 QTL regions for the $S c$ alleles that likely promote a fitness advantage while growing at $40{ }^{\circ} \mathrm{C}$ (SI Appendix, Table S7). Among these, IRA1, a regulator of the RAS pathway, was previously validated as a heat-QTL in OS3/OS104 crosses (34). Moreover, two additional genes involved in the RAS/cAMP signaling pathway ( $E S B 1$ and GPB2) were mapped in heat QTLs, supporting its involvement in in mediating heat resistance as previously suggested by Parts et al. (34).

The potential causal genes detected in $S$. cerevisiae genomes in $\mathrm{Sc} / \mathrm{Sj}, \mathrm{Sc} / \mathrm{Sk}$, and $\mathrm{Sc} / \mathrm{Se}$ hybrids may contain amino acid variants that are affecting protein function. Hence, we analyzed these genes through SIFT analysis (Sorting Intolerant From Tolerant) to identify nonsynonimous SNPs underlying the observed phenotypic difference between alleles $(43,44)$. SIFT analysis was carried out on the 82 predicted $S$. cerevisiae causal genes. A strong effect on the protein function was detected in $23 \%$ of potential causal genes due to amino acid differences between the $S$. cerevisiae parental strains (SI Appendix, Table S8), while ca. 38\% of mutations were inferred as tolerated, and for the remaining $39 \%$, no nonsynonymous SNPs at the protein-coding region were detected.

Gene ontology (GO) analysis did not help to narrow down choices of potential causal gene candidates, since the enrichment GO terms were, at a broad level, only generally associated with intracellular membrane-bound organelle, cytoplasm, catalytic activity, and cellular processes in all the conditions.

In total 14, 22, and 11 pleiotropic QTLs were mapped in $\mathrm{Sc} / \mathrm{Sj}$, $\mathrm{Sc} / \mathrm{Sk}$ and $\mathrm{Sc} / \mathrm{Se}$ hybrids, respectively (Dataset S3). A 7-kb region on the $S$. cerevisiae chromosome XIII was common across all conditions tested for $\mathrm{ScSj}_{\mathrm{m}}$ hybrids, but, interestingly, it was not detected for $\mathrm{Sc}_{\mathrm{m}} \mathrm{Sj}$ in any condition tested. This region contains the genes CLU1 (a subunit of eIF3), ANY1 (a protein involved in phospholipid flippase), and $H X T 2$ (a high-affinity glucose transporter). It is possible that the phenotypic effect of variation in these genes depends solely on mitochondrial-nuclear interactions, independent of the condition. CLU1 is known to play a role in mitochondrial distribution and morphology but it maintains its respiratory function and inheritance $(45,46)$. The $\Delta c l u 1$ mutants possess a more condensed mitochondrial mass found at one side of the cell (45). They are haplo insufficient in nutrient-limited media $(45,47)$ and haplo proficient in phosphorus-limited media (48).

In parallel to the comparison of small pools performed with Multipool, Pooled selection experiments were performed on $\mathrm{Sc}_{\mathrm{m}} / \mathrm{Se}$ and $\mathrm{Sc} / \mathrm{Se}_{\mathrm{m}}, \mathrm{Sc}_{\mathrm{m}} / \mathrm{Su}$ segregants. For the selected pools, the strength of selection was chosen such that $\sim 0.1 \%$ of the population survived the selective condition which was then pooled for comparison to the unselected population. Specifically, $\mathrm{Sc}_{\mathrm{m}} / \mathrm{Se}$ and $\mathrm{Sc} / \mathrm{Se}_{\mathrm{m}}$ were selected for a variety of selectable traits of industrial relevance, spanning from high maltose or glucose concentrations $(35 \%)$ to $\mathrm{H}_{2} \mathrm{O}_{2}$ treatment $(4 \mathrm{mM})$, and very low temperature $\left(4^{\circ} \mathrm{C}\right)$, while the $\mathrm{Sc}_{\mathrm{m}} \mathrm{Su}$ hybrid progeny were selected in yeast extract peptone dextrose (YPD) medium at high temperature $\left(40{ }^{\circ} \mathrm{C}\right)$ and YPD with levulinic acid $(50 \mathrm{mM})$, acetic acid $(0.35 \%)$ at $23{ }^{\circ} \mathrm{C}$.

The linkage analysis on the Pooled selection segregants yielded narrow intervals, averaging at $18.1 \mathrm{~kb}$ and $20 \mathrm{~kb}$ in $\mathrm{Sc} / \mathrm{Se}$ and $\mathrm{Sc}_{\mathrm{m}} / \mathrm{Su}$ hybrids, respectively. However, only a limited number of QTLs were identified, except for YP-Maltose (SI Appendix, Table S5). No QTLs were found among segregating $S$. uvarum alleles (SI Appendix, Table S9).

In total, 35 of the 41 QTL regions detected in $\mathrm{Sc}_{\mathrm{m}} / \mathrm{Se}$ and $\mathrm{Sc} /$ $\mathrm{Se}_{\mathrm{m}}$ segregants were present in hybrids with a fitness advantage in high maltose concentrations (SI Appendix, Table S9). However, it was unexpected that only one QTL was identified when selection took place at $4{ }^{\circ} \mathrm{C}$, given that in the Multipool analysis in the same condition, 45 QTLs were identified. Characteristics such as growth at low temperature, which, albeit selectable, do not show extremes phenotypes, are better discriminated using the Multipool approach as it exploits the richness of fitness data acquired through individual phenotyping. Growth at $4{ }^{\circ} \mathrm{C}$ may not be strong-enough selection (i.e., doesn't kill the majority of individuals in the population), resulting in less discrimination in the Pooled selection approach.

Similarly, Pooled selection of $\mathrm{Sc}_{\mathrm{m}} / \mathrm{Su}$ segregants yielded only nine intervals of interest, all mapping to $S$. cerevisiae alleles across the four conditions tested. These regions included a single interval conferring tolerance to acetic acid, four conferring selective advantage at high temperature, and four giving tolerance in an environment containing levulinic acid. As mentioned above, none were found in the $S$. uvarum genome.

As with Multipool QTL, we identified several genes, which had already been reported as having a phenotypic effect or a function closely linked to the condition tested. For $\mathrm{Sc}_{\mathrm{m}} / \mathrm{Se}$ and $\mathrm{Sc} / \mathrm{Se}_{\mathrm{m}}$ hybrids, we detected eight genes in the Pooled Segregants in high maltose concentrations, in which deletion was reported to cause osmotic stress sensitivity (SI Appendix, Table S10). Among these, $S K O 1$, a basic leucine zipper transcription factor mapped in $S$. eubayanus alleles of $\mathrm{Sc} / \mathrm{Se}_{\mathrm{m}}$ segregants, has been described having a major role in mediating HOG pathway-dependent osmotic regulation (49).

Overall, our results indicate that the Multipool approach with individuals at each extreme of a phenotype distribution is more efficient than a highly selected pool approach. However, the highly selected pool approach can identify rare genotypes of linked recombinant variants that are too rare to be in the arrays used to choose individuals for the Multipool approach.

Different Types of Mitochondria Have a Profound Effect on the QTL Landscape. Mitochondrial-nuclear interactions have been reported as having a major role in phenotypic variation both in intraspecies and interspecies yeast hybrids $(18,30,31,50)$, affecting respiration (29), fermentation properties $(18,51)$, progeny fitness $(52,53)$, reproductive isolation (50), and nuclear transcription (18). Given the complexity and the diversity of the hybrid background, thorough mapping of the epistasis in genome-wide studies 
has been a challenge. Here, in order to evaluate how the mitochondria inherited may affect the QTL landscape, we compared QTL regions mapped in diploid hybrid progeny derived from tetraploid lines harboring different mitochondria but the same parental nuclear inputs.

Interestingly, the majority of QTLs detected via the Multipool method were exclusive to a specific mitotype (Fig. 3). In fact, in all of the conditions analyzed, only ca. $2.45 \%$ QTL regions (the mean percentage for all hybrids in all the conditions) were in common among the segregants from tetraploid hybrids with different mitochondria. This difference in the QTL landscape between the same yeast hybrid cross, differing only for the mitotype, is consistent with the idea that mitochondrial-nuclear interactions have a genome-wide effect and are important in the context of evolution, as already demonstrated by several studies both in yeast $(50,54)$ and in other organisms $(55-57)$.

The experiments carried out via Pooled Selection also demonstrated mitotype-specific QTLs. Only one QTL region was in common in the $\mathrm{Sc} / \mathrm{Se}$ hybrids with different mitochondria: a 21-kb region on chromosome I, containing the genes FLO1 and $\mathrm{PHO11}$, where specific Sc alleles are associated with an increase in fitness at high maltose concentrations. Given these are located in a subtelomeric region, known to be highly variable in copy number and location in addition to sequence as well as difficult to assemble $(58,59)$, the causal genetic variation may be an unknown sequence linked to the FLO1 and PHO11 genes, as these regions are not assembled in all of the genomes utilized here.

Among the QTLs shared between $\mathrm{Sc}_{\mathrm{m}} \mathrm{Sj}$ and $\mathrm{ScSj}_{\mathrm{m}}$ segregants, a major maltose QTL near the telomeric regions of chromosome

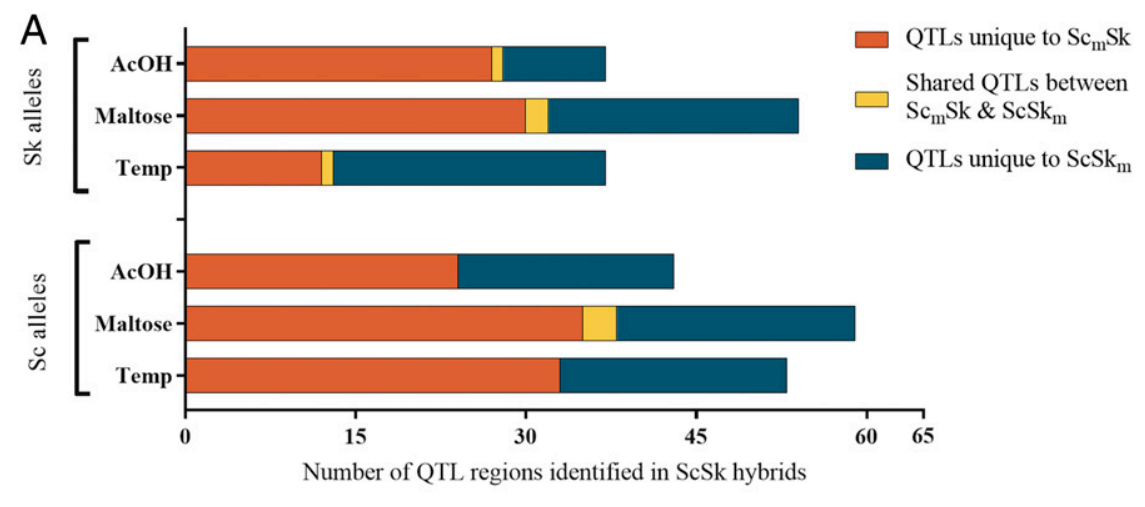

$\mathrm{B}$

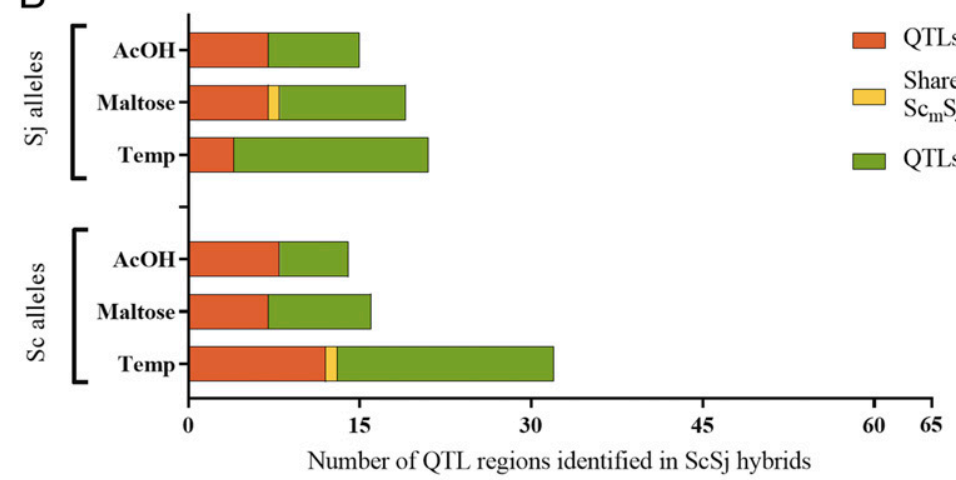

C

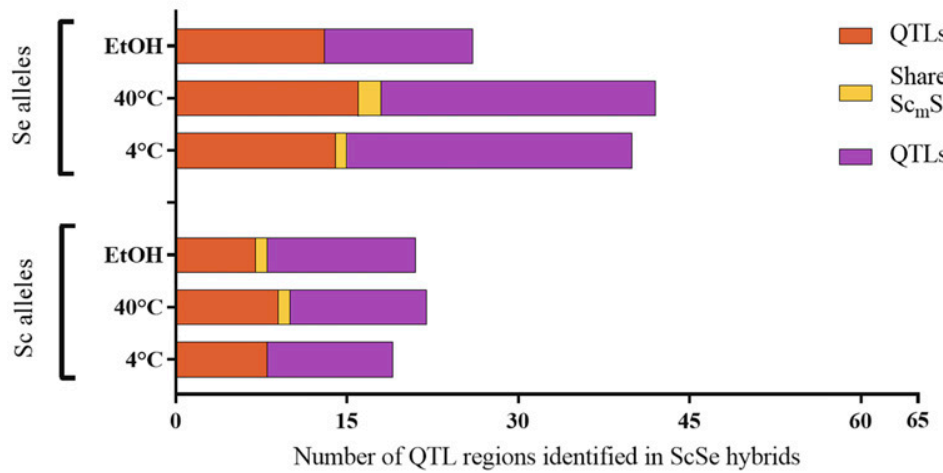

Fig. 3. Hybrids with different mitotype exhibit a different QTL landscape. Boxplot of the distribution of QTLs in S. cerevisiae/S. kudriavzevii (A), S. cerevisiae/ S. jurei $(B)$, and S. cerevisiae/S. eubayanus hybrids $(C)$. The QTL regions are grouped first by the alleles and then by growth condition in which they were identified: acetic acid (ACOH), high maltose concentrations (Maltose), low temperature $\left(16{ }^{\circ} \mathrm{C}\right.$ or $\left.4{ }^{\circ} \mathrm{C}\right)$, heat, and ethanol (EtOH). The small proportion of QTLs shared between mitotypes of the same hybrid is represented in yellow. 
II of $S$. cerevisiae ( 780 to $795 \mathrm{~kb}$ ) was detected with a high LOD score $(>17)$. The recurrence of this QTL and its high LOD score could be ascribed to the MAL3 multigene complex in the subtelomeric region and the natural variation in copy number, location, and sequence of this complex (58). Furthermore, this maltose QTL is also mapped on the $S$. cerevisiae alleles of both $\mathrm{Sc}_{\mathrm{m}} / \mathrm{Sk}$ and $\mathrm{Sc} / \mathrm{Sk}_{\mathrm{m}}$ hybrids, pointing to a more general effect of these allelic variants rather than a strain background dependent one.

Overlap of QTL Regions between Different Hybrids Facilitates the Identification of Causal Genes. One way to help to identify relevant genes within QTL regions, which are not specific to a particular hybrid combination, is to investigate overlapping QTL regions detected in different hybrids. Such an approach can lead to the unambiguous identification of genes underlying the phenotypic effect observed.

A total of 12 overlapping intervals, shared by at least three hybrid genomes, were mapped in low-temperature, high-maltose, and high-ethanol QTLs, while an additional 52 intervals were overlapping between two hybrids (Dataset S4). Within acetic acid QTLs, only 12 overlapping intervals were detected with no region shared between more than two species, suggesting a greater diversity of variants connected with the phenotype. Among these intervals, we identified several candidates with biological functions closely linked to the selection condition. For instance, a lowtemperature QTL mapped both in $S$. cerevisiae and $S$. kudriavzevii includes $O S H 6$ (Fig. 4A), related to sterol metabolism and, as such, membrane fluidity, often considered a feature of lowtemperature adaptation (60). Similarly, an acetic acid QTL mapped in both $\mathrm{Sc} / \mathrm{Sj}_{\mathrm{m}}$ and $\mathrm{Sc}_{\mathrm{m}} \mathrm{Sk}$ hybrids includes $N Q M 1$, a nuclear transaldolase involved in oxidative stress response, known to be induced by acetic acid stresses (61) (Fig. 4B).

Ethanol and high-temperature QTLs were analyzed only in $\mathrm{Sc} /$ Se hybrids, limiting the outcomes compared to the other traits. Nonetheless, we found three overlapping ethanol QTLs with a major region mapped on chromosome XV shared between genomes (Fig. 4C). Moreover, this region included the genes $P A C 1, V P H 1$, and MOD5 in which null mutations are linked to a decreased fitness in ethanol supplemented media $(62,63)$.

A major overlap was detected in the subtelomeric region of chromosome II, with a maltose QTL shared between the $S$. cerevisiae allele of all $\mathrm{Sc} / \mathrm{Sj}$ and $\mathrm{Sc} / \mathrm{Sk}$ hybrids and the $S$. jurei alleles of $\mathrm{Sc} / \mathrm{Sj}_{\mathrm{m}}$. The QTL contains a causal gene, MAL33, a MAL-activator protein, and two transporters, CTP1 and PHO89, involved in the transport of citrate and phosphate, respectively, which are key metabolites for the glycolytic pathway (Fig. 4E).

Remarkably, two low-temperature QTL regions were mapped in at least one mitotype of all tetraploid hybrids analyzed through Multipool. The overlapping regions resulted in both cases in a single-gene intersection with COQ6 (Fig. $4 F$ ) and PEP3 (Fig. 4D) identified in four and five different intervals, respectively. $C O Q 6$ is a mitochondrial monooxygenase, which, in addition to its known role in mitochondrial respiration, is involved in fatty acid $\beta$-oxidation (64). PEP3, instead, is a component of the CORVET membrane-tethering complex, and its role at cold temperature was previously suggested by large-scale competition studies (39). Moreover, SIFT analysis performed with mutfunc (65) predicted a strong deleterious effect of the SNP in the OS253 PEP3 variant, due to a substitution in a conserved region.

Validation of QTLs via Reciprocal Hemizygosis Analysis. The narrow mapping intervals identified in the $\mathrm{Sc} / \mathrm{Sk}_{\mathrm{m}}$ hybrid allowed singlegene studies to validate the effect of candidate alleles, which were not strongly identified as causal genes. The fitness of the allelic variants was tested with reciprocal-hemizygosity analysis (RHA) (25) performed on ASI2, FUS3, and GIT1, which are candidate QTLs in acetic acid, low temperature $\left(16^{\circ} \mathrm{C}\right)$, and high maltose, respectively (Fig. 5, $A$ and SI Appendix, Table S11).
Among the genes included in the acetic acid QTL, ASI2, part of the Asi ubiquitinase complex, was defined as a potential candidate gene, as its null mutant was previously described as sensitive to oxidative stress in systematic studies (66). FUS3, a MAPK protein, was previously described as haplo-insufficient in largescale competition studies at $16{ }^{\circ} \mathrm{C}$ (39). Lastly, we selected the plasma membrane permease GIT1 included in a high LOD QTL region in chromosome III identified at high maltose concentrations, along HMRA1, HMRA2, CDC39, CDC50, and OCA4. GIT1 was deemed the most-promising candidate, as it is involved in phosphate and glycerol-3-phospate transport, important metabolites of the glycolytic pathway (67).

The phenotypic effect of $A S I 2, F U S 3$, and GIT1 alleles were validated via RHA performed on the hemizygote tetraploid parents $\left(\mathrm{Sc}^{\mathrm{OS} 253} / \mathrm{Sk}^{\mathrm{OS} 575} / \mathrm{Sc}^{\mathrm{OS} 104} / \mathrm{Sk}^{\mathrm{IFO} 1802}\right)$ in $\mathrm{YPD}+0.3 \%$ acetic acid at $30{ }^{\circ} \mathrm{C}$, YPD at $10{ }^{\circ} \mathrm{C}$, and YP-Maltose $(15 \%)$ at $25^{\circ} \mathrm{C}$, respectively (Fig. 5, B-D and SI Appendix, Table S12). We observed a significant difference of the growth curve integral area (SI Appendix, Table S12) between the performance of the allelic variants in all the three genes tested, confirming their impact in the selection condition and validating them as having causal variant alleles. The FUS3 ${ }^{\mathrm{OS} 253}$ and $G I T 1^{\mathrm{OS} 104}$ alleles performed better than the FUS3 ${ }^{\mathrm{OS} 104}$ and $G I T 1^{\mathrm{OS} 253}$ alleles in terms of specific growth rate $(P$ value $=0.0010$ and 0.0335 , respectively) and integral area $(P$ value $=0.0047$ and 0.0435 , respectively) (SI Appendix, Table S12), mirroring what we have seen in our Multipool QTL screening. For the $A S I 2$ gene, the $A S I 2^{\mathrm{OS} 253}$ was the allele prevalent in the highfitness pool exposed to a high concentration of acetic acid. In the phenotypic validation, the $A S I 2^{\mathrm{OS} 253}$ variant performed worse than the $A S I 2^{\mathrm{OS} 104}$ in terms of integral area $(P$ value $=0.0479)$ and $\mathrm{T}_{\text {mid }}$ $(P$ value $=0.0182)$ but it reached a higher maximum biomass $(P$ value $=0.0001)$. Although the growth parameters for these two alleles are clearly different, it is more ambiguous whether their fitness performance overlaps with that one detected in the QTL study in which the $A S I 2^{\mathrm{OS} 253}$ was the allele prevalent in the highfitness pool. This discrepancy could be due to other genes in the close proximity, masking its effect, or to the difference in the phenotypic screening employed, as the F12 segregants were assayed for their growth in solid media. Many of yeast QTLs have shown to be environment and background dependent and have linked sets of quantitative trait nucleotides (QTN) (68-70). In fact, it has been shown that sporulation efficiency in yeast is controlled by four QTNs (71).

Interspecies Hybrids Generate New QTLs Not Present in Parental Intraspecies Crosses. Finally, we investigated whether the phenotypic effect of the ASI2, FUS3, and GIT1, allelic variants, was exclusive to the interspecies hybrid background. If the QTLs are hybrid dependent, then they should not be present in crosses involving only strains belonging to the same species. Hence, we evaluated the presence of QTLs involving different $S$. cerevisiae alleles in intraspecific crosses between the two relevant $S$. cerevisiae strains (Fig. 5, $E-H$ ). Specifically, the validation was performed through RHA on $S$. cerevisiae $\mathrm{Sc}^{\mathrm{OS} 104} / \mathrm{Sc}^{\text {OS253 }} \mathrm{dip}-$ loids. No significant difference in fitness between the allelic variants was observed for both ASI2 and GIT1 alleles, indicating that such QTLs are exclusive to interspecies hybrid background (SI Appendix, Table S12). In the $\mathrm{Sc}^{\mathrm{OS} 104} / \mathrm{Sc}^{\text {OS253 }}$ diploids, FUS3 alleles showed a significant difference in the integral area of the curve $(P$ value $=0.0196 ;$ SI Appendix, Table S12) and growth rate $(P$ value $=0.0014$, SI Appendix, Table S12). This phenotypic variation is, however, opposite to that one observed in $\mathrm{Sc} / \mathrm{Sk}_{\mathrm{m}}$ tetraploids, suggesting that also in this case the allelic differences are influenced by the interspecies hybrid genomes. Moreover, a temperature QTL including FUS3 was also mapped in $\mathrm{Sc} / \mathrm{Sj}_{\mathrm{m}}$ hybrids, suggesting that the phenotypic effect may be indeed hybrid background independent. 


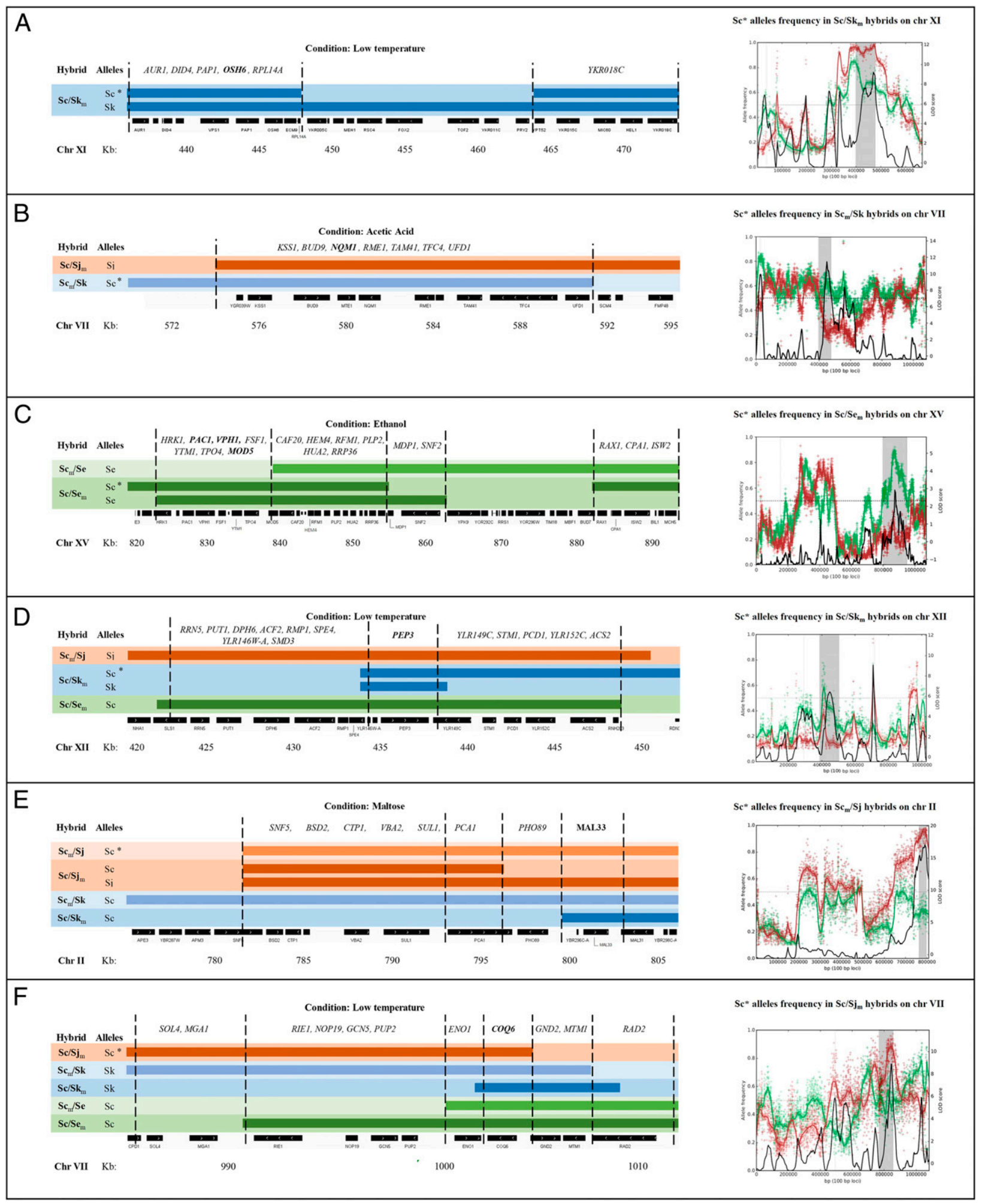

Fig. 4. Example of interspecies QTLs detected in S. cerevisiae/S. jurei, S. cerevisiae/S. kudriavzevii, and S. cerevisiae/S. eubayanus hybrids for low temperature $(A, D, F)$, acetic acid $(B)$, ethanol $(C)$ and maltose $(E)$ traits. The QTL regions, represented by colored bars, are mapped onto $S$. cerevisiae chromosomes to identify the overlapping QTL intervals, and the genes shared between different species and hybrids. The parental species alleles for each hybrid are stated, and the genes in bold denotes potential causal genes. The QTL plots represents the frequency of S. cerevisiae alleles, marked in the figure with an asterisk, from different hybrid pool lines. The red and green lines represent the alternative allele frequency of high- and low-fitness pools using the $\mathrm{Sc}^{\mathrm{OS} 104} \mathrm{genome}$ as reference. The black line indicates the LOD score, and the gray area is the $90 \%$ credible interval of the significance. The dash line is the threshold of LOD considered in this study. 


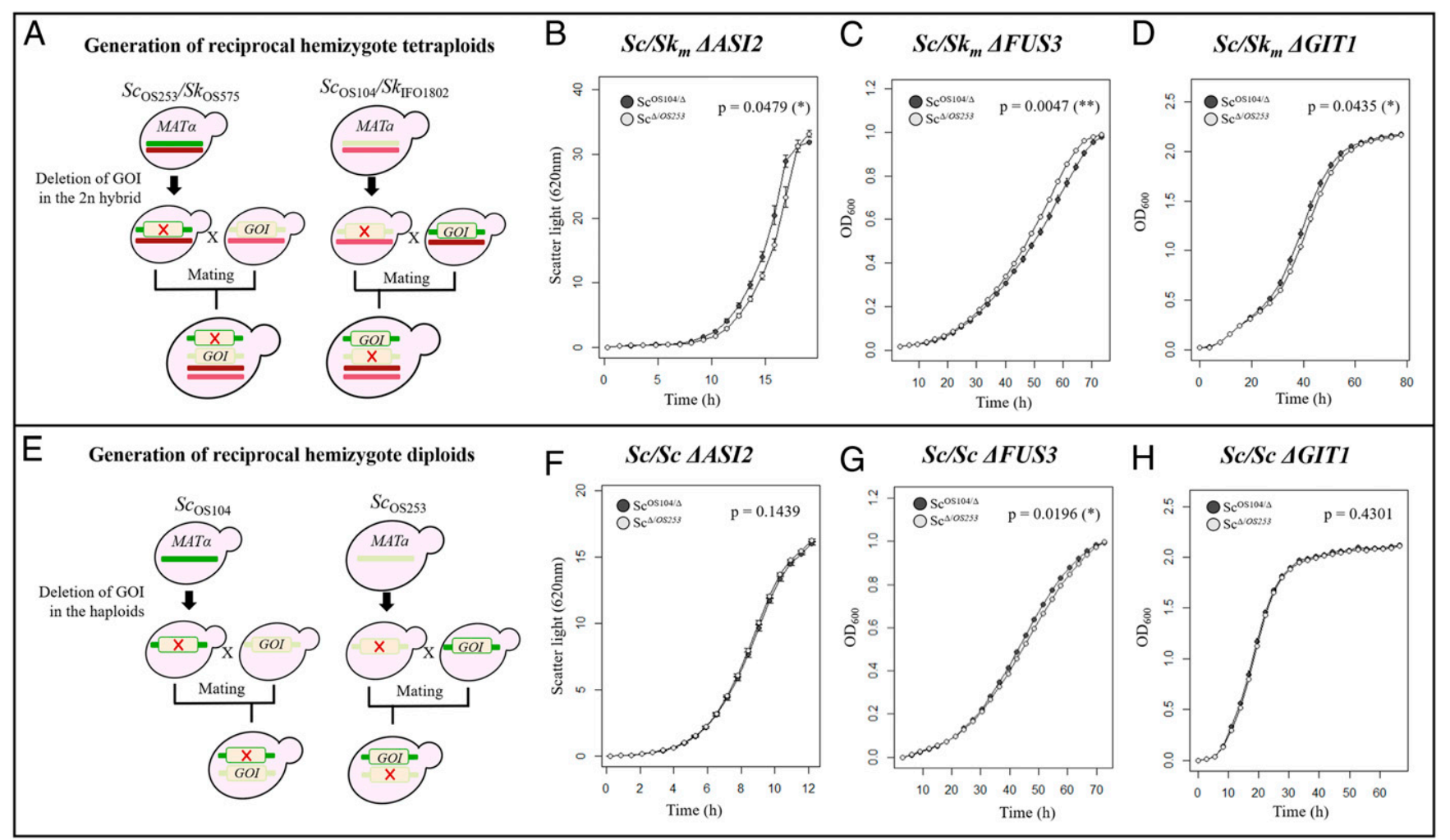

Fig. 5. Validation of the phenotypic effect of candidate genes in inter- and intraspecies hybrid background. Diagram of the construction of the reciprocal hemizygote $\mathrm{ScSk}_{\mathrm{m}}$ tetraploid strains $(A)$. The gene of interest (GOI) was first deleted from the respective ScSk diploid hybrids. The engineered $2 \mathrm{n}$ hybrids were crossed to construct the reciprocal hemizygote tetraploid strains. Growth curves of ScSk $\mathrm{m}_{\mathrm{m}}$ reciprocal hemizygotes for the ASI2, FUS3, and GIT1 genes are

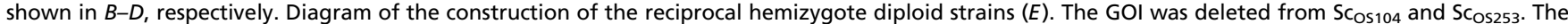
engineered haploid strains were crossed to construct the hemizygote diploids. Growth curves of SC OS104/OS253 reciprocal hemizygotes for the ASI2, FUS3, and GIT1 are shown in $F-H$, respectively. Fitness assays were performed in YPD supplemented with $0.3 \%$ acetic acid $(B$ and $F), Y P D$ at $10{ }^{\circ} \mathrm{C}(C$ and $G)$, and $Y P+$ maltose $15 \%(D$ and $H)$ as outlined in Materials and Methods. Significance difference between the integral area of the reciprocal hemizygotes is shown as $P$ value assessed by Student's $t$ test.

\section{Discussion}

Hybrid sterility can be overcome by doubling the genome of the hybrid. Such fertility-restored hybrids, known as amphidiploids, are commonly found in plants and represent the majority of major evolutionary events in angiosperms $(72,73)$. Tetraploidization, resulting in amphidiploids, can also restore fertility in yeast hybrids (22). Saccharomyces yeast hybrids have now entered the realm of classical genetic analysis as well as molecular genetic analysis. Previous studies have created variants of many interspecies hybrids, and some have even been able to complete one round of meiotic recombination, allowing some linkage analysis of genetic variants associated with traits of interest. Here, we take this one step further by overcoming hybrid sterility in ways that allow continuous sequential crossing resulting in advanced intercross lines that bring in the full power of breeding genetics and quantitative genetic analysis of hybrid traits. We demonstrate that multiple traits of interest can be analyzed with the same sensitivity and resolution as performed for intraspecies studies. Moreover, we compare different QTL analysis approaches and can advise that the Multipool approach is more efficient for detecting most QTLs than pools of highly selected subpopulations. The Multipool approach will not resolve tightly linked sets of QTN within a QTL, such as the $A S I 2$ alleles likely to be linked to other alleles of opposite phenotypic effect. In such cases the highly selected pool can identify these complex situations by enriching for rare recombinant haplotypes in the region (69). The identification of overlapping QTL regions among progeny from different tetraploid hybrids allowed us to focus on the genes that are more likely to be responsible for the phenotypic variation. Such an approach can also identify alleles which exert a background-independent effect.

With the sterility of hybrids overcome, we have shown that the hybrid situation is even more complex than the complex trait analysis within a species.

Firstly, the type of mitochondria inherited affect the QTL landscape. We were able to compare QTL regions mapped in diploid hybrid progeny derived from tetraploid parental lines with different mitochondria. Interestingly, the majority of QTLs detected were exclusive to a specific mitotype, and only a small number of QTL regions were in common among the segregants from tetraploid hybrids with different mitochondria. Although the extent of the mitochondrial-nuclear epistasis cannot be easily extrapolated from these data, these results reinforce the idea that genetic interactions between the mitochondrial and nuclear genome are important. Studies on interspecies yeast hybrids in the laboratory have shown a correlation between the origin of the mitochondrial genome and the higher stability of one of the nuclear genomes $(74,75)$. Moreover, several mitochondrial-nuclear incompatibilities leading to respiratory deficiencies have been identified in yeast hybrids (50), and some are associated with the splicing of mitochondrial intron cox1I $3 \beta$ (76). The incompatibility of the $S$. uvarum mitochondrion with the $S$. cerevisiae nucleus was reinforced by the transplacement of mitochondria isolated from $S$. uvarum $(77,78)$. A recent study on S.cerevisiae/S. uvarum hybrids has also shown that selective mitochondria retention is influenced by its contribution to hybrid fitness in different environments, and the type of mitochondria inherited affects the nuclear transcription 
at alleles level (18). In yeast, mitochondrial-nuclear epistasis also contributes to coadaptation to changing environmental conditions $(53,54,79,80)$. These mitochondrial-nuclear interactions are examples of the growing number of Bateson-Dobzhansky-Müller incompatibilities affecting the function of certain processes and fitness but are not directly leading to inviability/infertility, so-called speciation genes, which are quite rare in Saccharomyces (81). Mitochondrial-nuclear epistasis has been shown to affect phenotypes in several taxa. In insects, such as Drosophila and Callosobruchus, exchange of mitochondrial DNA variant has led to decreased metabolic rate (82) and shortened life span (83). In mice, it is known to affect cognition and respiratory functions $(84,85)$. Interactions between mitochondrial and nuclear genomes can result in cytoplasmic male sterility in plants (86) and impact aging and longevity in humans (87).

Secondly, and even more profound, new QTLs are generated in hybrids. This is allelic variation that has no phenotypic consequences in a parent species but has phenotypic consequences in the hybrid. RHA (25) on ASI2, FUS3, and GIT1 was carried out to assess the fitness the $\mathrm{Sc}^{\mathrm{OS} 253}$ and $\mathrm{Sc}^{\mathrm{OS} 104}$ alleles in the interspecific tetraploid hybrid $\left(\mathrm{Sc}^{\mathrm{OS} 253} / \mathrm{Sk}^{\mathrm{OS} 575} / \mathrm{Sc}^{\mathrm{OS} 104} / \mathrm{Sk}^{\mathrm{IFO} 1802}\right.$ ) and in the $S$. cerevisiae intraspecific diploid hybrid $\left(\mathrm{Sc}^{\mathrm{OS} 104} / \mathrm{Sc}^{\mathrm{OS} 253}\right)$, and we showed that these QTLs are exclusive to interspecies hybrid background. The potential, therefore, for exploiting natural genetic variation in developing new hybrids is greater than expected and bodes well for future advances in yeast breeding for improvement.

\section{Materials and Methods}

Strains, Growth Conditions, and Sporulation. The complete list of diploid strains used in this study is shown in Dataset S5. These strains were chosen for the creation of de novo interspecies and intraspecies hybrids. Yeast strains were routinely cultured in YPD medium (1\% yeast extract, $2 \%$ peptone, and $2 \%$ glucose, Formedium). To select for the drug resistance markers, YPD medium was supplemented either with $300 \mu \mathrm{g} / \mathrm{mL}$ geneticin, $300 \mu \mathrm{g} / \mathrm{mL}$ hygromycin $\mathrm{B}, 100 \mu \mathrm{g} / \mathrm{mL}$ nourseothricin, or $10 \mu \mathrm{g} / \mathrm{mL}$ bleomycin.

Construction of Genetically Stable Haploid Strains. All the haploid strains used in this study are listed in SI Appendix, Table S13. Genetically stable haploid S. cerevisiae strains used to make the hybrids were obtained from the Louis laboratory (88) and from derivatives of these (89). S. jurei haploid strain was constructed previously in Delneri laboratory (3). S. uvarum, S. eubayanus, and $S$. kudriavzevii haploid strains were engineered in this study, by deleting the $\mathrm{HO}$ gene. The plasmids and the primers used for the gene deletion and verification are listed in SI Appendix, Table S14 and Dataset S6, respectively. The methodological details for the generation of haploid strains are presented in SI Appendix, Supplementary Methods.

Generating Tetraploid Hybrids and Sporulation. Mass mating, sporulation, and tetrad dissection were conducted by following standard protocols (90). Two approaches to generate tetraploid hybrids were used (Fig. 1). The hybrid nature of the diploid spores was determined by species specific PCR (91), and the primers used are listed in Dataset S6. The detailed methodological procedure for hybrids construction is presented in SI Appendix, Supplementary Methods.

Multigenerational Advanced Intercross Lines. Tetraploids were subjected to sporulation, and tetrads were isolated for dissection to assess the spore viability and phenotypic variation and to prepare the spores for further rounds of mating. The methodological details for the construction of multigenerational hybrid lines are presented in SI Appendix, Supplementary Methods.

Analysis of Mitochondrial Origin in Hybrids. For each diploid mater, a petite version was generated by exposure to ethidium bromide (EtBr) (92). Isolates were seeded at an approximate density of 300 individuals per plate. A 3- $\mu \mathrm{L}$ drop of $\mathrm{EtBr}(10 \mathrm{mg} / \mathrm{mL})$ was spotted onto the center of each plate. A ring around the spot formed where all cells were killed due to the toxic effects of EtBr. Surrounding this kill zone, a ring of petite colonies form. Loss of mitochondria enables petites to grow faster than colonies with functional mitochondria in the presence of EtBr. These individuals were confirmed as petites by their inability to grow on YPD plates containing ethanol and glycerol, nonfermentable carbon sources (93). The specific mitotype was identified by amplifying the COX2 and COX3 genes through colony PCR as described previously (18). The primers used for the amplification of these genes are listed in Dataset S6.

Phenotypic Assays. A high-throughput spot assay was performed using Singer ROTOR HDA robot (Singer Instruments, United Kingdom) as mentioned previously (2). The fitness of $\sim 384$ hybrid spores was assessed at five different temperatures (i.e., 10, 16, 23, 30, and $37^{\circ} \mathrm{C}$ ) under different carbon sources at $30{ }^{\circ} \mathrm{C}$ (i.e., YPA $+10 \% \& 15 \%$ maltose, YPA $+10 \%$ \& $15 \%$ fructose, YPA $+10 \%$ $\& 15 \%$ sucrose, YPA $+10 \% \& 15 \%$ galactose, and YPA $+30 \% \& 35 \%$ glucose) and under different environmental stressors at $30{ }^{\circ} \mathrm{C}$ (i.e., YPAD $+6 \%$ \& $10 \%$ ethanol, YPAD $+0.3 \% \& 0.5 \%$ acetic acid, YPAD $+4 \mathrm{mM}$ hydrogen peroxide, YPAD + $50 \mathrm{mM}$ levulinic acid, and YPA + 10\% \& 15\% glycerol).

Fitness analysis was done following two different strategies, using either Phenosuite software (Singer Instruments) or the PHENOS platform (94). Details of the phenotypic analysis are given in SI Appendix, Supplementary Methods.

Sequencing, Mapping, and Variant Calling. Each parental genome in the hybrid was sequenced previously (2) or in this study at the Earlham Institute or at the Genomic Technologies Core Facility of the University of Manchester. Two different pooling strategies were followed for Multipool and Pooled selection, respectively. In strategy one, from each selective media plate, the top 20 performing F12 individuals, with the highest fitness, and the 20 lowest performing F12 individuals were picked for pooling. In second strategy, a pool of $1 \times 10^{8} \mathrm{~F} 12$ cells were seeded onto each selection condition as well as the YPD control. Selection conditions were prepared so that only the top 0.1 to $1 \%$ of the pool would be capable of growth.

The sequencing was done to 100 to $120 \times$ coverage on the Illumina platform

Paired-end raw Illumina sequence reads were quality checked through FastQC 0.11 .5 (95) and trimmed through Trimmomatic 0.36 (96). The complete variant calling pipeline is displayed in SI Appendix, Fig. S4 and described in SI Appendix.

QTL Mapping. Two different strategies for detection of QTLs have been followed for the Multipool and for Pooled selection, respectively. The detailed methodological pipeline for both approaches is given in SI Appendix Supplementary Methods. QTL regions associated with the phenotype were identified by analyzing the changes in frequencies of SNP alleles across the genomes. The QTL intervals, gene content, and LOD scores are included in Dataset S2.

GO and SIFT Analysis. GO terms were determined using the GO Term Finder tool of Saccharomyces Genome Database with the Bonferroni correction for multiple hypothesis and a $P$ value cutoff of 0.01 .

Potential causal genes were analyzed with the SIFT algorithm to assess whether amino acid variants were predicted to influence the protein function. SIFT analysis were conducted using data from Bergström et al. (44) on the S. cerevisiae strains OS3, OS104, and OS253 and the tool mutfunc (65).

Validation of Candidate Genes through RHA. The candidate genes selected for RHA were chosen based on their LOD score and on GO studies carried out with YeastMine (97) and Funspec (98) to prioritize terms connected to the phenotypic trait tested.

We performed RHA (25) on the selected genes ASI2, FUS3, and GIT1. PCRmediated deletion of the target genes was performed in the engineered $S$. cerevisiae/S. kudriavzevii hybrid (Sc ${ }^{\mathrm{OS} 253} / \mathrm{Sk}^{\mathrm{OS} 575}$ and $\mathrm{Sc}^{\mathrm{OS} 104} / \mathrm{Sk}^{\mathrm{IF01802}}$ ) and on S. cerevisiae haploid strains $\left(\mathrm{Sc}^{\mathrm{OS} 253}\right.$ and $\mathrm{Sc}^{\mathrm{OS} 104}$ ) to delete the $\mathrm{S}$. cerevisiae allele in each of them. Tetraploid interspecific reciprocal hemizygotes were constructed by mating the two diploid S. cerevisiae/S. kudriavzevii hybrids (Fig. 5A). Diploid intraspecific reciprocal hemizygotes were constructed by mating the two S. cerevisiae strains (Fig. $5 E$ ). The strains constructed for RHA are listed in SI Appendix, Table S15. The fitness of the $S$. cerevisiae allelic variants of FUS3, GIT1, and ASI2 was assessed both in the hemizygous $S$. cerevisiae/S. kudriavzevii tetraploids and in the hemizygous $S$. cerevisiae diploids. The methodological details for the construction of the strains and fitness analysis are presented in the SI Appendix, Supplementary Methods.

Data Availability. Sequencing data have been deposited in the European Nucleotide Archive (https://www.ebi.ac.uk/ena/browser/view/PRJEB44105) (99). All other study data are included in the article and/or supporting information.

ACKNOWLEDGMENTS. We wish to thank Gianni Liti, Chris Powell, Philippe Malcorps, and Stewart Wilkinson for useful discussions and lan Donaldson for initial bioinformatic support. Sequencing was done through Genomic Services at the Earlham Institute and Genomic Technologies Core Facility at 
the University of Manchester. This work was funded by a Biotechnology and Biological Sciences Research Council (BBSRC) grant to E.J.L. (BB/L022508/1) and D.D. (BB/L21471/1) in collaboration with SAB-Miller and AB-InBev. F.V. is supported by H2020-MSCA-ITN-2017 grant to D.D. (764364; https://cordis. europa.eu/project/id/764364). A.H.R. has been supported by a BBSRC Collaborative Awards in Science and Engineering studentship to E.J.L. (BB/L017229/ 1). Strains were obtained from various culture collections as referenced. The

1. J. Mallet, Hybrid speciation. Nature 446, 279-283 (2007).

2. S. Naseeb et al., Whole genome sequencing, de Novo assembly and phenotypic profiling for the new budding yeast species Saccharomyces jurei. G3 (Bethesda) 8 2967-2977 (2018)

3. S. Naseeb et al., Saccharomyces jurei sp. nov., isolation and genetic identification of a novel yeast species from Quercus robur. Int. J. Syst. Evol. Microbiol. 67, 2046-2052 (2017).

4. G. Liti, D. B. Barton, E. J. Louis, Sequence diversity, reproductive isolation and species concepts in Saccharomyces. Genetics 174, 839-850 (2006).

5. G. Liti, A. Peruffo, S. A. James, I. N. Roberts, E. J. Louis, Inferences of evolutionary relationships from a population survey of LTR-retrotransposons and telomericassociated sequences in the Saccharomyces sensu stricto complex. Yeast 22, 177-192 (2005)

6. G. Liti, E. J. Louis, Yeast evolution and comparative genomics. Annu. Rev. Microbiol. 59, 135-153 (2005).

7. S. S. González, E. Barrio, A. Querol, Molecular characterization of new natural hybrids of Saccharomyces cerevisiae and S. kudriavzevii in brewing. Appl. Environ. Microbiol. 74, 2314-2320 (2008)

8. K. Lopandic, Saccharomyces interspecies hybrids as model organisms for studying yeast adaptation to stressful environments. Yeast 35, 21-38 (2018)

9. B. Dunn, G. Sherlock, Reconstruction of the genome origins and evolution of the hybrid lager yeast Saccharomyces pastorianus. Genome Res. 18, 1610-1623 (2008).

10. D. Libkind et al., Microbe domestication and the identification of the wild genetic stock of lager-brewing yeast. Proc. Natl. Acad. Sci. U.S.A. 108, 14539-14544 (2011).

11. S. S. González, E. Barrio, J. Gafner, A. Querol, Natural hybrids from Saccharomyces cerevisiae, Saccharomyces bayanus and Saccharomyces kudriavzevii in wine fermentations. FEMS Yeast Res. 6, 1221-1234 (2006).

12. B. Gallone et al., Interspecific hybridization facilitates niche adaptation in beer yeast. Nat. Ecol. Evol. 3, 1562-1575 (2019)

13. R. Barbosa et al., Evidence of natural hybridization in Brazilian wild lineages of Saccharomyces cerevisiae. Genome Biol. Evol. 8, 317-329 (2016).

14. T. Snoek et al., Large-scale robot-assisted genome shuffling yields industrial Saccharomyces cerevisiae yeasts with increased ethanol tolerance. Biotechnol. Biofuels 8, 32 (2015).

15. K. Krogerus, F. Magalhães, V. Vidgren, B. Gibson, New lager yeast strains generated by interspecific hybridization. J. Ind. Microbiol. Biotechnol. 42, 769-778 (2015).

16. S. Mertens et al., A large set of newly created interspecific Saccharomyces hybrids increases aromatic diversity in lager beers. Appl. Environ. Microbiol. 81, 8202-8214 (2015).

17. E. García-Ríos et al., Improving the cryotolerance of wine yeast by interspecific hybridization in the genus Saccharomyces. Front. Microbiol. 9, 3232 (2019).

18. S. K. Hewitt et al., Plasticity of mitochondrial DNA inheritance and its impact on nuclear gene transcription in yeast hybrids. Microorganisms 8, 494 (2020).

19. M. Lairón-Peris et al., Differential contribution of the parental genomes to a S. cerevisiae $\times S$. uvarum hybrid, inferred by phenomic, genomic, and transcriptomic analyses, at different industrial stress conditions. Front. Bioeng. Biotechnol. 8, 129 (2020).

20. E. M. Piatkowska, S. Naseeb, D. Knight, D. Delneri, Chimeric protein complexes in hybrid species generate novel phenotypes. PLoS Genet. 9, e1003836 (2013).

21. K. Lopandic et al., Genotypic and phenotypic evolution of yeast interspecies hybrids during high-sugar fermentation. Appl. Microbiol. Biotechnol. 100, 6331-6343 (2016).

22. D. Greig, R. H. Borts, E. J. Louis, M. Travisano, Epistasis and hybrid sterility in Saccharomyces. Proc. Biol. Sci. 269, 1167-1171 (2002).

23. K. Schwartz, J. W. Wenger, B. Dunn, G. Sherlock, APJ1 and GRE3 homologs work in concert to allow growth in xylose in a natural Saccharomyces sensu stricto hybrid yeast. Genetics 191, 621-632 (2012).

24. A. Zimmer et al., QTL dissection of Lag phase in wine fermentation reveals a new translocation responsible for Saccharomyces cerevisiae adaptation to sulfite. PLoS One 9, e86298 (2014).

25. L. M. Steinmetz et al., Dissecting the architecture of a quantitative trait locus in yeast Nature 416, 326-330 (2002)

26. M. Okuno et al., Next-generation sequencing analysis of lager brewing yeast strains reveals the evolutionary history of interspecies hybridization. DNA Res. 23, 67-80 (2016).

27. E. Baker et al., The genome sequence of Saccharomyces eubayanus and the domestication of lager-brewing yeasts. Mol. Biol. Evol. 32, 2818-2831 (2015).

28. I. Masneuf, J. Hansen, C. Groth, J. Piskur, D. Dubourdieu, New hybrids between Sac charomyces sensu stricto yeast species found among wine and cider production strains. Appl. Environ. Microbiol. 64, 3887-3892 (1998)

29. W. Albertin et al., The mitochondrial genome impacts respiration but not fermentation in interspecific Saccharomyces hybrids. PLoS One 8, e75121 (2013).

30. E. P. Baker et al., Mitochondrial DNA and temperature tolerance in lager yeasts. Sci. Adv. 5, eaav1869 (2019).
S. eubayanus type strain, CBS12357, was obtained from the culture collection Centraal Bureau voor de Statistiek (CBS) at the Koninklijke Nederlandse Akademie van Wetenschappen (KNAW; Westerdijk Fungal Biodiversity Institute) under Material Transfer Agreement (MTA) for academic research use. The Chinese strains LZSP32.1, CDFM212.1, and 48BYC-4 were obtained from Prof. Fen-Yang Bai and the China General Microbiological Culture Collection Center under MTA for academic research use.

31. X. C. Li, D. Peris, C. T. Hittinger, E. A. Sia, J. C. Fay, Mitochondria-encoded genes contribute to evolution of heat and cold tolerance in yeast. Sci. Adv. 5, eaav1848 (2019)

32. M. D. Edwards, D. K. Gifford, High-resolution genetic mapping with pooled sequencing. BMC Bioinformatics 13, S8 (2012)

33. I. M. Ehrenreich et al., Dissection of genetically complex traits with extremely large pools of yeast segregants. Nature 464, 1039-1042 (2010).

34. L. Parts et al., Revealing the genetic structure of a trait by sequencing a population under selection. Genome Res. 21, 1131-1138 (2011).

35. B. R. Gibson, S. J. Lawrence, J. P. Leclaire, C. D. Powell, K. A. Smart, Yeast responses to stresses associated with industrial brewery handling. FEMS Microbiol. Rev. 31, 535-569 (2007)

36. C. Belloch, S. Orlic, E. Barrio, A. Querol, Fermentative stress adaptation of hybrids within the Saccharomyces sensu stricto complex. Int. J. Food Microbiol. 122, 188-195 (2008).

37. J. M. Cherry et al., Saccharomyces Genome Database: The genomics resource of budding yeast. Nucleic Acids Res. 40, D700-D705 (2012).

38. G. Hueso et al., A novel role for protein kinase $\mathrm{Gcn} 2$ in yeast tolerance to intracellular acid stress. Biochem. J. 441, 255-264 (2012).

39. C. M. Paget, J. M. Schwartz, D. Delneri, Environmental systems biology of coldtolerant phenotype in Saccharomyces species adapted to grow at different temperatures. Mol. Ecol. 23, 5241-5257 (2014).

40. M. C. Teixeira, L. R. Raposo, N. P. Mira, A. B. Lourenço, I. Sá-Correia, Genome-wide identification of Saccharomyces cerevisiae genes required for maximal tolerance to ethanol. Appl. Environ. Microbiol. 75, 5761-5772 (2009).

41. H. Sinha et al., Sequential elimination of major-effect contributors identifies additional quantitative trait loci conditioning high-temperature growth in yeast. Genetics 180, 1661-1670 (2008).

42. F. A. Cubillos et al., High-resolution mapping of complex traits with a four-parent advanced intercross yeast population. Genetics 195, 1141-1155 (2013).

43. P. Kumar, S. Henikoff, P. C. Ng, Predicting the effects of coding non-synonymous variants on protein function using the SIFT algorithm. Nat. Protoc. 4, 1073-1081 (2009)

44. A. Bergström et al., A high-definition view of functional genetic variation from natural yeast genomes. Mol. Biol. Evol. 31, 872-888 (2014).

45. S. D. Fields, M. N. Conrad, M. Clarke, The S. cerevisiae CLU1 and D. discoideum cluA genes are functional homologues that influence mitochondrial morphology and distribution. J. Cell Sci. 111, 1717-1727 (1998).

46. K. S. Dimmer et al., Genetic basis of mitochondrial function and morphology in Saccharomyces cerevisiae. Mol. Biol. Cell 13, 847-853 (2002).

47. P. Pir et al., The genetic control of growth rate: A systems biology study in yeast. BMC Syst. Biol. 6, 4 (2012)

48. D. Delneri et al., Identification and characterization of high-flux-control genes of yeast through competition analyses in continuous cultures. Nat. Genet. 40, 113-117 (2008)

49. M. Rep et al., The Saccharomyces cerevisiae Sko1p transcription factor mediates HOG pathway-dependent osmotic regulation of a set of genes encoding enzymes implicated in protection from oxidative damage. Mol. Microbiol. 40, 1067-1083 (2001).

50. H. Y. Lee et al., Incompatibility of nuclear and mitochondrial genomes causes hybrid sterility between two yeast species. Cell 135, 1065-1073 (2008).

51. L. Solieri, O. Antúnez, J. E. Pérez-Ortín, E. Barrio, P. Giudici, Mitochondrial inheritance and fermentative: Oxidative balance in hybrids between Saccharomyces cerevisiae and Saccharomyces uvarum. Yeast 25, 485-500 (2008)

52. C. Zeyl, Experimental evolution with yeast. FEMS Yeast Res. 6, 685-691 (2006)

53. C. Zeyl, B. Andreson, E. Weninck, Nuclear-mitochondrial epistasis for fitness in Saccharomyces cerevisiae. Evolution 59, 910-914 (2005).

54. J. F. Wolters et al., Mitochondrial recombination reveals mito-mito epistasis in yeast. Genetics 209, 307-319 (2018).

55. C. K. Ellison, O. Niehuis, J. Gadau, Hybrid breakdown and mitochondrial dysfunction in hybrids of Nasonia parasitoid wasps. J. Evol. Biol. 21, 1844-1851 (2008)

56. J. A. Mossman, J. G. Tross, N. Li, Z. Wu, D. M. Rand, Mitochondrial-nuclear interactions mediate sex-specific transcriptional profiles in drosophila. Genetics 204, 613-630 (2016)

57. R. I. Wernick et al., Sex and mitonuclear adaptation in experimental Caenorhabditis elegans populations. Genetics 211, 1045-1058 (2019).

58. G. Liti et al., Population genomics of domestic and wild yeasts. Nature 458, 337-341 (2009).

59. E. J. Louis, M. M. Becker, Subtelomeres (Springer Berlin Heidelberg, Berlin, Heidelberg, 2014).

60. P. Buzzini, E. Branda, M. Goretti, B. Turchetti, Psychrophilic yeasts from worldwide glacial habitats: Diversity, adaptation strategies and biotechnological potential. FEMS Microbiol. Ecol. 82, 217-241 (2012)

61. S. Michel, M. A. Keller, M. M. Wamelink, M. Ralser, A haploproficient interaction of the transaldolase paralogue NQM1 with the transcription factor VHR1 affects stationary phase survival and oxidative stress resistance. BMC Genet. 16, 13 (2015). 
62. F. van Voorst, J. Houghton-Larsen, L. Jønson, M. C. Kielland-Brandt, A. Brandt Genome-wide identification of genes required for growth of Saccharomyces cerevisiae under ethanol stress. Yeast 23, 351-359 (2006).

63. K. Yoshikawa et al., Comprehensive phenotypic analysis for identification of genes affecting growth under ethanol stress in Saccharomyces cerevisiae. FEMS Yeast Res. 9, 32-44 (2009).

64. A. M. Awad et al., Coenzyme $\mathrm{Q}_{10}$ deficiencies: Pathways in yeast and humans. Essays Biochem. 62, 361-376 (2018).

65. O. Wagih et al., A resource of variant effect predictions of single nucleotide variants in model organisms. Mol. Syst. Biol. 14, e8430 (2018).

66. V. J. Higgins et al., Phenotypic analysis of gene deletant strains for sensitivity to oxidative stress. Yeast 19, 203-214 (2002).

67. Y. Popova, P. Thayumanavan, E. Lonati, M. Agrochão, J. M. Thevelein, Transport and signaling through the phosphate-binding site of the yeast Pho84 phosphate transceptor. Proc. Natl. Acad. Sci. U.S.A. 107, 2890-2895 (2010).

68. F. A. Cubillos et al., Assessing the complex architecture of polygenic traits in diverged yeast populations. Mol. Ecol. 20, 1401-1413 (2011).

69. G. Liti, E. J. Louis, Advances in quantitative trait analysis in yeast. PLoS Genet. 8 e1002912 (2012).

70. H. Sinha, B. P. Nicholson, L. M. Steinmetz, J. H. McCusker, Complex genetic interactions in a quantitative trait locus. PLoS Genet. 2, e13 (2006).

71. J. Gerke, K. Lorenz, B. Cohen, Genetic interactions between transcription factors cause natural variation in yeast. Science 323, 498-501 (2009).

72. X. Wang et al., The genome of the mesopolyploid crop species Brassica rapa. Nat Genet. 43, 1035-1039 (2011).

73. X. Zhang et al., Interspecific hybridization, polyploidization, and backcross of Brassica oleracea var. alboglabra with B. rapa var. purpurea morphologically recapitulate the evolution of Brassica vegetables. Sci. Rep. 6, 18618 (2016).

74. Z. Antunovics, H. V. Nguyen, C. Gaillardin, M. Sipiczki, Gradual genome stabilisation by progressive reduction of the Saccharomyces uvarum genome in an interspecific hybrid with Saccharomyces cerevisiae. FEMS Yeast Res. 5, 1141-1150 (2005).

75. D. Peris et al., Synthetic hybrids of six yeast species. Nat. Commun. 11, 2085 (2020).

76. M. Špírek, S. Poláková, K. Jatzová, P. Sulo, Post-zygotic sterility and cytonuclear compatibility limits in S. cerevisiae xenomitochondrial cybrids. Front. Genet. 5, 454 (2015).

77. M. Osuský, J. Kissová, L. Kovác, Interspecies transplacement of mitochondria in yeasts. Curr. Genet. 32, 24-26 (1997).

78. M. Spírek, A. Horváth, J. Piskur, P. Sulo, Functional co-operation between the nucle of Saccharomyces cerevisiae and mitochondria from other yeast species. Curr. Genet. 38, 202-207 (2000).

79. S. Paliwal, A. C. Fiumera, H. L. Fiumera, Mitochondrial-nuclear epistasis contributes to phenotypic variation and coadaptation in natural isolates of Saccharomyces cerevisiae. Genetics 198, 1251-1265 (2014).

80. M. De Chiara et al., Discordant evolution of mitochondrial and nuclear yeast genomes at population level. BMC Biol. 18, 49 (2020).
81. B. A. Dujon, E. J. Louis, Genome diversity and evolution in the budding yeasts (Saccharomycotina). Genetics 206, 717-750 (2017)

82. D. K. Dowling, K. C. Abiega, G. Arnqvist, Temperature-specific outcomes of cytoplasmic-nuclear interactions on egg-to-adult development time in seed beetles. Evolution 61, 194-201 (2007).

83. C. T. Zhu, P. Ingelmo, D. M. Rand, GXGXE for lifespan in Drosophila: Mitochondrial, nuclear, and dietary interactions that modify longevity. PLoS Genet. 10, e1004354 (2014).

84. P. L. Roubertoux et al., Mitochondrial DNA modifies cognition in interaction with the nuclear genome and age in mice. Nat. Genet. 35, 65-69 (2003).

85. A. M. Betancourt et al., Mitochondrial-nuclear genome interactions in non-alcoholic fatty liver disease in mice. Biochem. J. 461, 223-232 (2014)

86. J. Hu et al., Mitochondria and cytoplasmic male sterility in plants. Mitochondrion 19 Pt B, 282-288 (2014).

87. G. J. Tranah, Mitochondrial-nuclear epistasis: Implications for human aging and longevity. Ageing Res. Rev. 10, 238-252 (2011).

88. F. A. Cubillos, E. J. Louis, G. Liti, Generation of a large set of genetically tractable haploid and diploid Saccharomyces strains. FEMS Yeast Res. 9, 1217-1225 (2009).

89. H. Louvel, A. Gillet-Markowska, G. Liti, G. Fischer, A set of genetically diverged Sac charomyces cerevisiae strains with markerless deletions of multiple auxotrophic genes. Yeast 31, 91-101 (2014)

90. F. Sherman, J. Hicks, Micromanipulation and dissection of asci. Methods Enzymol. 194 21-37 (1991).

91. A. Muir, E. Harrison, A. Wheals, A multiplex set of species-specific primers for rapid identification of members of the genus Saccharomyces. FEMS Yeast Res. 11, 552-563 (2011).

92. E. S. Goldring, L. I. Grossman, J. Marmur, Petite mutation in yeast. II. Isolation of mutants containing mitochondrial deoxyribonucleic acid of reduced size. J. Bacteriol. 107, 377-381 (1971)

93. M. Day, Yeast petites and small colony variants: For everything there is a season. $A d v$. Appl. Microbiol. 85, 1-41 (2013).

94. D. B. H. Barton et al., PHENOS: A high-throughput and flexible tool for microorganism growth phenotyping on solid media. BMC Microbiol. 18, 9 (2018).

95. S. Andrews, FastQC A Quality Control Tool for High Throughput Sequence Data. Brabaham Bioinformatics. https://www.bioinformatics.babraham.ac.uk/projects/ fastqd . Deposited 8 March 2016.

96. A. M. Bolger, M. Lohse, B. Usadel, Trimmomatic: A flexible trimmer for Illumina sequence data. Bioinformatics 30, 2114-2120 (2014).

97. R. Balakrishnan et al., YeastMine-An integrated data warehouse for Saccharomyces cerevisiae data as a multipurpose tool-kit. Database (Oxford) 2012, bar062 (2012).

98. M. D. Robinson, J. Grigull, N. Mohammad, T. R. Hughes, FunSpec: A web-based cluster interpreter for yeast. BMC Bioinformatics 3, 35 (2002).

99. I. Donaldson et al., QTL traits in fertile inter-specific yeast hybrids. European Nucleotide Archive (ENA). https://www.ebi.ac.uk/ena/browser/view/PRJEB44105. Deposited 18 May 2021 